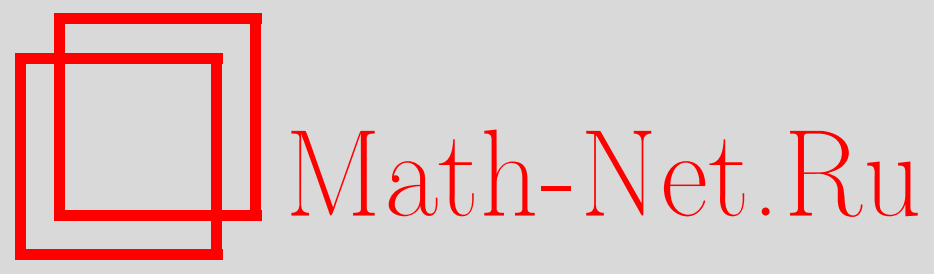

Г. Килибарда, В. Б. Кудрявцев, Ш. М. Ушчумлич, Коллективы автоматов в лабиринтах, Дискрет. матем., 2003, том 15, выпуск 3, 3-39

DOI: https://doi.org/10.4213/dm203

Использование Общероссийского математического портала Math-Net.Ru подразумевает, что вы прочитали и согласны с пользовательским соглашением http: //www.mathnet.ru/rus/agreement

Параметры загрузки:

IP : 54.224 .60 .19

26 апреля 2023 г., 18:12:07

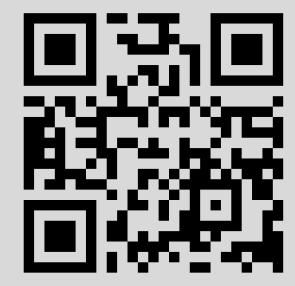


УДК 519.95

\title{
Коллективы автоматов в лабиринтах
}

\author{
○ 2003 г. Г. Килибарда, В.Б.Кудрявцев, Ш. Ушчумлич
}

Поведение автоматов в лабиринтах - сравнительно новое направление теории автоматов, по тематике которого имеется уже более ста публикаций. В работе дается обзор исследований по одному из разделов этого направления, поведению коллективов автоматов в лабиринтах. Выделяются основные понятия, проблематика, достижения, методы решения задач и открытые проблемы, касаюшиеся коллективов автоматов. Основные утверждения в ряде случаев приводятся в более сильном виде по сравнению с их первоначальными формулировками.

\section{1. Введение}

Статья является продолжением обзорной статьи [54], посвященной поведению независимых систем автоматов в лабиринтах. Как выяснилось, независимая система автоматов оказывается слишком слабой при решении значительной части задач, возникающих в этой области. Поэтому предлагались различные усиления этой модели вплоть до модели машины Тьюринга (в ее обобшенном виде). Поскольку машина Тьюринга легко решает рассматриваемые данным направлением задачи, то самым интересным и главным усијением модели независимой системы автоматов оказывается коллектив автоматов. В данной статье дается обзор понятий, задач и результатов, касающихся коллективов автоматов в лабиринтах.

В отличие от независимых систем автоматов, коллектив автоматов анализирует лабиринты с учетом положения его членов в лабиринте. Коллективы автоматов могут быть снабжены камнями, играюшими роль ограниченной внешней памяти для коллективов. Камень - это флажок-метка, который может быть или на руках у любого автомата, или в некоторой вершине обследуемого коллективом лабиринта. Автомат может забирать или оставлять камень в вершине, в которой оказался, но общее количество камней у данного коллектива является постоянным. Камни можно рассматривать также как автоматы простейшего вида, перемещение которых полностью определяется другими автоматами коллектива. В $[18,19]$ установлено, что коллектив из одного автомата и одного камня не может обойти все конечкые плоские мозаичные лабиринты; в [4] показано, что коллектив из одного автомата и двух камней решает эту задачу, при этом отмечено, что коллектив из двух автоматов также решает эту задачу. Наряду с этим в [12] и [25] показано, что класс указанных лабиринтов допускает естественное расслоение такое, что для любого его слоя найдется коллектив из одного автомата с камнем, обходящий этот слой; в качестве параметра расслоения выступает число дыр в лабиринте.

Аналогичный вопрос для класса всех конечных и бесконечных плоских мозаичных лабиринтов исследуется в работах $[3,10,23,36,51]$. В них установлены некоторые 
простейшие по числу автоматов и камней коллективы, обходящие все такие лабиринты. В работах $[23,51]$ практически завершено описание всех таких коллективов (открытой проблемой остался только случай коллектива, состоящего из одного автомата и 4 камней); в этих же работах приведено решение указанной задачи для лабиринтов, не содержащих бесконечных дыр.

Для лабиринтов более общего вида в $[3,15]$ доказано наличие ловушки уже в трехмерном случае. В [52] установлено наличие бесконечной трехмерной ловушки сразу для всех коллективов автоматов, при этом коллективы остаются в шаре ограниченного радиуса в этой ловушке. Подобные результаты оказываются верными и в планарном случае, для лабиринтов, имеющих вид кубического графа [32].

Специальными классами лабиринтов являются так называемые сигнатурные лабиринты и лабиринты Савича. Для первого вида лабиринтов в [67] получены описания простейших коллективов автоматов с камнями, находящих специальную вершину в этих лабиринтах. Для второго вида лабиринтов установлено, что проблема выхода из них по специальным путям эквивалентна открытой проблеме совпадения языков, распознаваемых детерминированными и недетерминированными линейно ограниченными машинами Тьюринга, что свидетельствует о больших потенциальных трудностях тематики [33, 34].

Начато исследование задачи о встрече коллективов автоматов в лабиринтах. Одним из возможных толкований этой задачи может быть описание для заданного класса лабиринтов всех пар коллективов, которые встречаются в любом лабиринте из этого класса. В [39] показано, что два коллектива, каждый из которых состоит из одного автомата и двух камней, могут решить задачу о встрече на плоскости, а в [44] указаны простейшие типы коллективов автоматов, решающих задачу о встрече на прямой и в плоскости.

В работах $[45,46]$ рассмотрен вариант задачи о встрече двух автоматов, находящихся в отношении хищник-жертва, где автомат-хищник пытается догнать автомат-жертву, а автомат-жертва стремится убежать от него; взаимодействие происходит в квадратном лабиринте. Приводятся условия, при которых указанная встреча происходит. В работах $[6,7,44]$ и других рассматривались возможности более общих моделей автоматов в лабиринтах. Так, например, в [7] показано, что автомат с магазинной памятью не может обойти все лабиринты, имеющие вид кубических графов, а в [44] приведены примеры автоматов со счетчиками, со стеками и магазинами, обходящих мозаичную плоскость. Установлено также, что существует автомат с магазином, который обходит любой конечный плоский односвязный шахматный лабиринт и останавливается после его обхода [6].

В работах многих авторов изучалась задача анализа для автоматов и лабиринтов (см., например, $[2,8,20,21,22,24,28,29,30,33,34])$. Эта задача состоит для заданного коллектива автоматов в описании всех лабиринтов, которые обходятся этим коллективом при возможных дополнительных соглашениях типа требования остановки после обхода. Попытки описать эти лабиринты в виде алгебры Клини встретили затруднения [20]; трудности возникли также при выяснении отношений между классами лабиринтов, представляющих решение задачи анализа для заданных коллективов автоматов [22]. В [2] показано, что классы лабиринтов, обходимые автоматами с камнями, неограниченно возрастают с увеличением числа камней. Анализу свойств нагруженных графов посвящена работа [63], в которой устанавливается, с какой сложностью может быть решена задача эквивалентности поведения автоматов в таких графах. В $[28,31]$ устанавливается связь между классами лабиринтов и формальными языками, что приводит к переплетению проблематики и переходу к решению задач для автоматов в лабиринтах и для языков.

Как видно из перечисленных результатов, основная проблематика для автоматов в лабиринтах группируется вокруг задач, условно называемых задачами синтеза и анализа. 
Задача синтеза состоит в описании тех автоматов и коллективов автоматов, которые обходят лабиринты из заданного класса. Главными объектами здесь выступают конечные автоматы с камнями, коллективы автоматов, автоматы с магазинной памятью и другие, a также конечные или бесконечные плоские мозаичные лабиринты, различные классы таких лабиринтов (как, например, лабиринты ограниченной связности, специальной геометрической формы), плоские, но не мозаичные, мозаичные в пространстве, конечные и бесконечные лабиринты и их подклассы и обобщения. В случае, когда для класса лабиринтов отсутствуют автоматы заданного типа, обходящие эти лабиринты, возникают такие задачи, как выделение тех лабиринтов, в которых автоматы не решают задачу обхода, то есть лабиринтов-ловушек или просто ловушек, и нахождение среди этих ловушек таких, которые обладают специальными свойствами, например, являются простейшими.

Задача анализа, как уже было сказано выше, состоит в описании по заданному автомату или коллективу автоматов всех лабиринтов или всех лабиринтов определенного вида, которые обходятся этими автоматами. В качестве автоматов и лабиринтов выступают объекты, указанные в задаче синтеза. Продвижение в решении этой задачи по сравнению с задачей синтеза значительно скромнее и состоит, по существу, в построении различных позитивных и негативных примеров возможных соответствий между конкретными множествами лабиринтов и автоматов, обходящих в точности эти лабиринты, или же отсутствие таковых.

К числу задач, примыкаюших к указанным задачам синтеза и анализа, относится поиск конкретных целей в лабиринтах, например, специальных областей или других автоматов; установление определенных свойств лабиринтов, например, наличие специальных шиклов, сильной связности и прочее. Сюда же могут быть отнесены и проблемы распознавания свойств геометрических изображений, допускающих описание с помощью формальных языков.

В качестве главной модели выступают конечные автоматы в конечных плоских мозаичных и бесконечных плоских мозаичных лабиринтах.

В данной работе сначала излагаются результаты по поведению коллективов автоматов в вышеприведенных классах лабиринтов и в некоторых их обобщениях, затем результаты, связанные с более общими моделями автоматов в лабиринтах. Задачи анализа даются в отдельном разделе.

Настоящая статья является развитием положений, приведенных авторами в работах $[27,56]$. Даются новые результаты, расширяется и уточняется ситуация, описанная в этих статьях.

\section{2. Основные понятия}

В данной статье мы пользуемся обозначениями и понятиями, введенными в [54]. Другими словами, мы исходим из того, что читателю известны понятия лабиринта, (прямоугольного, целочисленного, мозаичного, правильного, шахматного) $n$-мерного лабиринта, некоторых специальных классов лабиринтов, например, класса змеевидных $n$-мерных лабиринтов, понятия дыры (преграды), Z-дыры, как и понятия лабиринтного монстра, независимой системы автоматов и т. п.

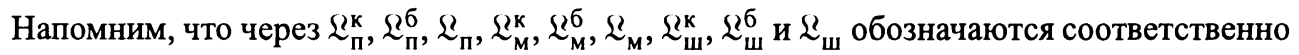
классы всех конечных, всех бесконечных и всех (конечных и бесконечных) прямоугольных плоских лабиринтов и соответствующие подклассы мозаичных и шахматных плоских лабиринтов. 
В [54] рассмотрено поведение независимой системы автоматов в лабиринтах. Здесь рассмотрим более сильный вариант поведения системы автоматов $\mathscr{A}$ в лабиринтах множества $\mathscr{L}(\Omega, \Sigma)$. Автоматы системы $\mathscr{A}$, двигаясь по любому лабиринту $L \in \widetilde{\mathscr{L}}(\Omega, \Sigma)$, будут учитывать, кроме метки вершины, в которой оказались, и всех меток дуг, исходящих из нее, еще и состояния всех других автоматов системы, которые оказались в той же самой вершине.

Систему автоматов $\mathscr{A}=\left\langle\mathfrak{U}_{q_{1}}^{1}, \ldots, \mathfrak{A}_{q_{n}}^{n}\right\rangle$, где $\mathfrak{U}_{q_{i}}^{i}=\left(A_{i}, Q_{i}, B_{i}, \varphi_{i}, \psi_{i}, q_{i}\right), 1 \leqslant i \leqslant n$, назовем $(\Omega, \Sigma)$-коллективом автоматов, если выполнены следующие условия:

(1) входной алфавит автомата $\mathfrak{A}_{q_{i}}^{i}=\left(A_{i}, Q_{i}, B_{i}, \varphi_{i}, \psi_{i}, q_{i}\right), 1 \leqslant i \leqslant n$, состоит из букв a вида $\left(\omega, \stackrel{*}{q}, \Sigma^{\prime}\right)$, где $\omega \in \Omega$ и $\Sigma^{\prime} \subseteq \Sigma$, а $\stackrel{*}{q}: \bar{n} \rightarrow \bigcup_{k=1}^{n} Q_{k}-$ частичная функция из множества индексов $\bar{n}_{*}=\{1, \ldots, n\}$ в множество $\bigcup_{k=1}^{n} Q_{k}$, не определенная на $i$, и такая, что значение $\stackrel{*}{q(j)}$ или не определено, или принадлежит $Q_{j}$ дляг любого $1 \leqslant j \leqslant n, j \neq i$

(2) $B_{i}=\Sigma \cup\{\theta\}$ для любого $1 \leqslant i \leqslant n$, причем $\theta$ - фиксированный элемент, не принадлежащий $\Sigma$;

(3) $\psi_{i}(q, a) \in \mathbf{p}_{3}(a) \cup\{\theta\}$ для любых $a \in A_{i}$ и $q \in Q_{i}$.

Интерпретируем функционирование коллектива $\mathscr{A}=\left\langle\mathfrak{U}_{q_{1}}^{1}, \ldots, \mathfrak{A}_{q_{n}}^{n}\right\rangle$ в некотором лабиринте $L_{v_{1}, \ldots, v_{n}} \in \tilde{\mathscr{L}}(\Omega, \Sigma)$ его движением в лабиринте $L_{v_{1}, \ldots, v_{n}}$ следующим образом. Автомат $\mathfrak{U}_{q_{i}}^{i}, 1 \leqslant i \leqslant n$, в начальный момент помещаем в вершину $v_{i} \in V(L)$, из которой он начинает свое движение в $L$. Предположим, что в некоторый момент $t \in \mathbf{N}_{0}$ этот автомат оказался в вершине $v_{i}^{l} \in V(L)$ и в состоянии $q_{i}^{l} \in Q_{i}$; при этом считаем, что $v_{i}^{0}=v_{i}$ и $q_{i}^{0}=q_{i}$ для любого $1 \leqslant i \leqslant n$. В следующий момент $t+1$ он перемещается в вершину $v_{i}^{t+1} \in V(L)$ и переходит в состояние $q_{i}^{t+1} \in Q_{i}$ согласно следующей процедуре. Он обозревает нагруженную звезду, образованную исходящими из этой вершины дугами. Его входной буквой $a_{i_{*}}^{t}$ в этот момент является тройка, образованная меткой вершины $v_{i}^{t}$, частичной функцией ${ }_{i}^{t}: \bar{n} \rightarrow \bigcup_{i=1}^{n} Q_{i}$ такой, что $\stackrel{*}{q}_{i}^{t}(j)=q_{j}^{t}$, если $v_{j}^{t}=v_{i}^{t}$ и $j \neq i$, и $\stackrel{*}{q}_{i}^{t}(j)$ не определено в противном случае, и множеством меток дуг, исходящих из вершины $v_{i}^{t}$. Функцию $\stackrel{*}{q}_{i}^{t}(j)$ можно описать набором вида $\left({ }^{i} w_{1}^{t}, \ldots,{ }^{i} w_{n}^{t}\right)$, где ${ }^{i} w_{j}^{t}=\stackrel{*}{q}_{i}^{t}(j)$ для всех $j$, для которых значение ${ }^{*}{ }_{i}^{t}(j)$ определено, и ${ }^{i} w_{j}^{t}=\theta^{\prime}$ в противном случае, где $\theta^{\prime}$ - некоторый фиксированный символ, не принадлежащий множеству $\bigcup_{i=1}^{n} Q_{i}$. Тогда, если $\psi_{i}\left(q_{i}^{t}, a_{i}^{t}\right) \neq \theta$, то вершиной $v_{i}^{t+1}$ будет вершина, в которую ведет в $L$ дуга из $v_{i}^{t}$ с меткой $\psi_{i}\left(q_{i}^{t}, a_{i}^{t}\right)$, а если $\psi_{i}\left(q_{i}^{t}, a_{i}^{t}\right)=\theta$, то автомат остается на месте, то есть $v_{i}^{t+1}=v_{i}^{t+1}$, и в обоих случаях $q_{i}^{t+1}=\varphi_{i}\left(q_{i}^{t}, a_{i}^{t}\right)$. Поскольку, существование вершины $v_{i}^{t+1}$ обеспечено условием 3, то этот процесс продолжается бесконечно. Таким образом, автомат $\mathfrak{U}_{q_{i}}^{i}$ осуществляет движение по лабиринту, последовательно проходя некоторый путь. Последовательность $\left(q_{i}^{0}, v_{i}^{0}\right),\left(q_{i}^{1}, v_{i}^{1}\right), \ldots$ называется поведением автомата $\mathfrak{A}_{q_{i}}^{i}$ из $(\Omega, \Sigma)$-коллектива $\mathscr{A}$ в лабиринте $L_{v_{1}, \ldots . v_{n}}$; при этом говорим, что $\mathfrak{A}_{q_{i}}^{i}$ обходит вершины $v_{i}^{0}, v_{i}^{1}, \ldots$, и обозначаем множество этих вершин через $\operatorname{Int}\left(\mathscr{A}, L_{v_{1}, \ldots, v_{n}} ; i\right)$. Последовательность $\pi\left(\mathscr{A}, L_{v_{1}, \ldots, v_{n}}\right)=\left(q_{1}^{0}, \ldots, q_{n}^{0}, v_{1}^{0}, \ldots, v_{n}^{0}\right),\left(q_{1}^{1}, \ldots, q_{n}^{1}, v_{1}^{1}, \ldots, v_{n}^{1}\right), \ldots$ такая, что для любого $i \in \bar{n}$ последовательность $\left(q_{i}^{0}, v_{i}^{0}\right),\left(q_{i}^{1}, v_{i}^{1}\right), \ldots$ является поведением автомата $\mathfrak{A}_{q_{i}}^{i}$ коллектива $\mathscr{A}$ в лабиринте $L_{v_{1}, \ldots, v_{n}}$, называется поведением $(\Omega, \Sigma)$-коллектива $\mathscr{A}$ в лаби- 
ринте $L_{v_{1}, \ldots . v_{n}}$. Введем обозначение

$$
\operatorname{Int}\left(\mathscr{A}, L_{v_{1}, \ldots . v_{n}}\right)=\bigcup_{i=1}^{n} \operatorname{Int}\left(\mathfrak{A}_{q_{i}}, L_{v_{i}} ; i\right)
$$

а также

$$
\operatorname{Fr}\left(\mathscr{A}, L_{v_{1}, \ldots, v_{n}}\right)=V\left(L_{v_{1}, \ldots, v_{n}}\right) \backslash \operatorname{Int}\left(\mathfrak{A}_{q_{i}}, L_{v_{1}, \ldots, v_{n}}\right) .
$$

$\operatorname{Ecли~} \operatorname{Int}\left(\mathscr{A}, L_{v_{1}, \ldots, v_{n}}\right)=V$, то говорим, что $\mathscr{A}$ обходит $L_{v_{1}, \ldots, v_{n}} ;$ в противном случае $L_{v_{1}, \ldots, v_{n}}$ является ловушкой для $\mathscr{A}$. Лабиринт $L$ называем сильной ловушкой для $\mathscr{A}$, если для любых $v_{1}, \ldots, v_{n}$ из $V(L)$ лабиринт $L_{v_{1}, \ldots . v_{n}}$ является ловушкой для $\mathscr{A}$.

Ясно, что лабиринтный монстр $\mathfrak{M}_{\mathfrak{A}}$, определенный такой системой, является примером лабиринтного $\mathfrak{L}$-монстра для любого $\mathfrak{L} \subseteq \widetilde{L}(\Omega, \Sigma)$. Его допустимость по отношению к лабиринтам множества $\mathfrak{L}$ следует из условия 3. В последующем, если будем говорить о коллективе автоматов $\mathscr{A}$, допустимом для $\mathfrak{L}$, или просто о коллективе автоматов $\mathscr{A}$ в $\mathfrak{L}$, то на самом деле будем иметь в виду монстр $\mathfrak{M}_{\mathfrak{A}}$.

Говорим, что коллектив $\mathscr{A}=\left\langle\mathfrak{A}_{q_{1}}^{1}, \ldots, \mathfrak{A}_{q_{n}}^{n}\right\rangle$, допустимый для $\tilde{\mathscr{L}}(\Omega, \Sigma)$, является синхронизированным в лабиринте $L \in \mathscr{L}(\Omega, \Sigma)$, если все автоматы коллектива $\mathscr{A}$ помещены в начальный момент в одну и ту же вершину, то есть рассматривается поведение коллектива $\not A$ в лабиринте $L_{f}$, где $f$ таково, что $f(i)=v_{0}$ для некоторой вершины $v_{0} \in V(L)$ для всех $1 \leqslant i \leqslant n$; в таком случае вместо записи $L_{v_{1}, \ldots, v_{n}}$ пишем $L_{v_{0}}$ или $\left(L ; v_{0}\right)$. Коллектив $\mathscr{A}$ сильно обходит неинициальный лабиринт $L$, если для любого $v \in V(L)$ коллектив $\mathscr{A}$ обходит лабиринт $L_{v}$.

Пусть $\mathscr{A}=\left\langle\mathfrak{A}_{1}, \ldots, \mathfrak{A}_{m}\right\rangle$, где $\mathfrak{A}_{i}=\left(A_{i}, Q_{i}, B_{i}, \varphi_{i}, \psi_{i}, q_{i}^{0}\right)$ для любого $i \in \bar{m},-$ некоторый $(\Omega, \Sigma)$-коллектив автоматов. Подсистема $\left\langle\mathfrak{A}_{i_{1}}, \ldots, \mathfrak{A}_{i_{k}}\right\rangle, \quad 1 \leqslant i_{1}<\ldots<i_{k} \leqslant m$, системы $\mathscr{A}$ называется системой автоматов-камней или просто системой камней в коллективе $\mathscr{A}$, если имеют место следующие условия:

(a) у автомата $\mathfrak{A}_{i_{j}}, 1 \leqslant j \leqslant k$, только одно состояние $q_{i_{j}}^{0}$;

(b) если для некоторого входа $a=\left(\omega, \stackrel{*}{q}, \Sigma^{\prime}\right)$ автомата $\mathfrak{A}_{i j}, 1 \leqslant j \leqslant k$, имеет место равенство $\psi_{i}(q, a)=\sigma \neq \theta, \sigma \in \Sigma^{\prime}$, то существует $l, 1 \leqslant l \leqslant m$, удовлетворяющее

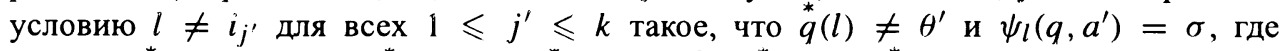
$a^{\prime}=\left(\omega, \stackrel{*}{q}^{\prime}, \Sigma^{\prime}\right)$, причем $\stackrel{*}{q}^{\prime}(l)=\theta^{\prime}, \stackrel{*}{q}^{\prime}\left(i_{j}\right)=q_{i_{j}}^{0}$ и $\stackrel{*}{q}^{\prime}\left(k^{\prime}\right)=\stackrel{*}{q}\left(k^{\prime}\right)$ для всех $1 \leqslant k^{\prime} \leqslant n$, $k^{\prime} \neq i_{j}, k^{\prime} \neq l$.

В случае, когда такая система камней существует, говорим, что у коллектива $\mathscr{A}$ есть система камней мошности $k$. Коллектив $\mathscr{A}=\left\langle\mathfrak{A}_{1}, \ldots, \mathfrak{A}_{m}\right\rangle$, у которого есть система камней мощности $k$, называется коллективом типа $(m-k, k)$. Ясно, что тип для конкретного коллектива неоднозначно определен. Если $k$ такое, что в $\mathscr{A}$ сушествует система камней мощности $k$ и не существует система камней мощности $k+1$, то рангом коллектива $\mathscr{A}$ называется число $m-k$.

Легко понять, что для соответствующего лабиринтного монстра $\mathfrak{M}_{\mathfrak{A}}$ любой автоматкамень представляет собою просто обыкновенный вершинный камень.

Понятие вершинного камня можно ввести и другим способом (см. [54]). Под системой камней понимаем множество (и даже мультимножество) флажков (маркеров, меток). Јюбой из этих маркеров-камней может быть или на руках у некоторого автомата данного коллектива, или в некоторой вершине рассматриваемого лабиринта. Такое распределение камней задается соответствующей функцией, и эта функция меняется только локально в 
вершинах, в которых оказывается хотя бы один камень и хотя бы один из членов коллектива. Автоматы коллектива могут забирать или оставлять камни, но их общее количество остается постоянным. Такая система камней может служить ограниченной внешней памятью для коллектива автоматов. Легко удостовериться, что коллектив из $p$ автоматов и $q$ камней-маркеров можно легко смоделировать с помощью некоторого коллектива автоматов типа $(p, q)$, и наоборот, любой коллектив автоматов типа $(p, q)$ моделируется некоторым коллективом из $p$ автоматов и $q$ камней-маркеров. Поэтому в вопросах, о которых идет речь в работе, эти два подхода к понятию камня, маркерный и автоматный, являются равносильными.

Рассмотрим теперь самый важный для нас случай - случай коллективов автоматов, допустимых для класса всех $n$-мерных лабиринтов. Рассмотрим этот случай более подробно (отличия в обозначениях здесь являются несущественными).

Коллектив автоматов $\mathscr{A}=\left\langle\mathfrak{U}_{1}, \ldots, \mathfrak{A}_{m}\right\rangle$, где $\mathfrak{U}_{i}=\left(A_{i}, Q_{i}, B_{i}, \varphi_{i}, \psi_{i}, \hat{q}_{i}\right)$ для любого $i \in \bar{m}$, является коллективом автоматов в $\mathbf{R}^{n}$, если для любого $i \in \bar{m}$ выполнены следующие условия:

(1) $A_{i}=\mathfrak{B}\left(\mathbf{D}_{n}\right) \times Q_{1} \times Q_{2} \times \ldots \times Q_{i-1} \times\{\theta\} \times Q_{i+1} \times \ldots \times Q_{m}$;

(2) $B_{i}=\mathbf{D}_{n} \cup\{\theta\}$;

(3) $\psi_{i}(q, a) \in \mathbf{p}_{1}(a) \cup\{\theta\}$ для любых $q \in Q_{i}$ и $a \in A$;

здесь $\theta-$ фиксированный элемент, не принадлежащий множеству $\mathbf{D}_{n} \cup \bigcup_{i=1}^{n} Q_{i}$.

Пусть $L$ - некоторый $n$-мерный лабиринт, $\vec{v}=\left(v_{1}, \ldots, v_{m}\right)$ - некоторый набор вершин длины $m$ этого лабиринта и $\vec{q}=\left(q_{1}, \ldots, q_{m}\right)$ - некоторый набор состояний автоматов коллектива $\mathscr{A}$ такой, что $q_{i} \in Q_{i}$ для любого $i \in \bar{m}$. Введем обозначение

$$
\left.a_{i}(\vec{v}, \vec{q})=\left(\left[v_{i}\right]_{L},\left[a_{i}(\vec{v}, \vec{q})\right)\right]_{1}, \ldots,\left[a_{i}(\vec{v}, \vec{q})\right]_{m}\right),
$$

где

$$
\left[a_{i}(\vec{v}, \vec{q})\right]_{j}= \begin{cases}q_{j}, & \text { если } v_{j}=v_{i} \text { и } j \neq i, \\ \theta, & \text { если } v_{j} \neq v_{i} \text { или } j=i .\end{cases}
$$

Пусть $\vec{v}_{0}=\left(v_{1}^{0}, v_{2}^{0}, \ldots, v_{m}^{0}\right)$ - некоторый набор длины $m$ вершин $n$-мерного лабиринта $L$. Под поведением коллектива $\mathscr{A}$ в $L_{\vec{v}_{0}}$ подразумеваем последовательность

$$
\pi\left(\mathscr{A} ; L, \vec{v}_{0}\right)=\left(\vec{v}_{0}, \vec{q}_{0}\right), \ldots,\left(\vec{v}_{t}, \vec{q}_{t}\right), \ldots
$$

такую, что для любых $i \in \bar{m}$ и $t \in \mathbf{N}_{0}$ выполнены следующие условия:

(а) $\vec{v}_{t}=\left(v_{1}^{\ell}, \ldots, v_{m}^{t}\right)$ и $\vec{q}_{t}=\left(q_{1}^{t}, \ldots, q_{m}^{t}\right)$;

(b) $q_{i}^{0}=\hat{q}_{i}$ и $q_{i}^{t+1}=\varphi_{i}\left(q_{i}^{t}, a_{i}\left(\vec{v}_{t}, \vec{q}_{t}\right)\right)$;

(c) если $\psi_{i}\left(q_{i}^{t}, a_{i}\left(\vec{v}_{t}, \vec{q}_{t}\right)\right) \neq \theta$, то $v_{i}^{t+1}$ такое, что $\left|\left(v_{i}^{t}, v_{i}^{t+1}\right)\right|=\psi_{i}\left(q_{i}^{t}, a_{i}\left(\vec{v}_{t}, \vec{q}_{t}\right)\right)$;

(d) если $\psi_{i}\left(q_{i}^{t}, a_{i}\left(\vec{v}_{t}, \vec{q}_{t}\right)\right)=\theta$, то $v_{i}^{t+1}=v_{i}^{t}$.

Введем также обозначения

$$
\operatorname{Int}\left(\mathscr{A} ; L, \vec{v}_{0}\right)=\bigcup_{i=0}^{\infty}\left(\bigcup_{j=1}^{m}\left\{v_{j}^{i}\right\}\right),
$$

$\operatorname{Fr}\left(\mathscr{A} ; L, \vec{v}_{0}\right)=V \backslash \operatorname{Int}\left(\mathscr{A} ; L, \vec{v}_{0}\right)$. 
Если в данных определениях $v_{1}^{0}=\ldots=v_{m}^{0}=v_{0}$ (то есть имеется в виду случай синхронизированного коллектива автоматов), то в последующем всегда будем говорить не о поведении $\mathscr{A}$ в $\left(L ; \vec{v}_{0}\right)$, а о поведении $\mathscr{A}$ в $n$-мерном лабиринте $\left(L ; v_{0}\right)$, и во всех введенных обозначениях, где фигурирует $\vec{v}_{0}$, будем писать $v_{0}$ вместо $\vec{v}_{0}$.

Говорим, что коллектив автоматов $\mathscr{A}$ обходит $n$-мерный лабиринт $\left(L ; v_{0}\right)$, если $\operatorname{Int}\left(\mathscr{A} ; L, v_{0}\right)=V(L) ;$ коллектив автоматов $\mathscr{A}$ сильно обходит (неинициальный) $n$-мерный лабиринт $L$, если для любого $v \in V(L)$ коллектив $\mathscr{A}$ обходит $(L ; v) ; n$-мерный лабиринт $\left(L ; v_{0}\right)$ является ловушкой для коллектива $\mathscr{A}$, если $\mathscr{A}$ не обходит $\left(L ; v_{0}\right)$. Примем также следующие соглашения: $n$-мерный лабиринт $\left(L ; v_{0}, v_{1}\right)$ является ловушкой для $\mathscr{A}$, если $v_{1} \in \operatorname{Fr}\left(\mathscr{A} ; L, v_{0}\right)$; (неинициальный) $n$-мерный лабиринт $L$ называется сильной синхронной ловушкой для коллектива автоматов $\mathscr{A}$, если для любого $v \in V(L)$ коллектив $\mathscr{A}$ не обходит $(L, v)$.

Пусть $\mathfrak{Q}-$ некоторый класс лабиринтов. На множестве всех пар $(i, j) \in \mathbf{N} \times \mathbf{N}_{0}$ определим частичный порядок $\leqslant$, полагая, что $(a, b) \leqslant(c, d)$ тогда и только тогда, когда $a \leqslant c$ и $b \leqslant d$. Будем писать $(c, d)<(a, b)$, если $(c, d) \leqslant(a, b)$ и $(c, d) \neq(a, b)$. Предикат $\mathbf{P}_{\mathfrak{Q}}(i, j)$ на множестве $\mathbf{N} \times \mathbf{N}_{0}$ определим таким образом, что $\mathbf{P}_{\mathfrak{Q}}(a, b)=1$, если существует коллектив типа $(a, b)$, обходящий все лабиринты из $\mathfrak{L}$, и $\mathbf{P}_{\mathfrak{Q}}(a, b)=0$, если такого коллектива не существует. Предикат $\mathbf{P}_{\mathfrak{Z}}$ назовем предикатом обхода (класса 2). Нетрудно видеть, что для любых целочисленных неотрицательных пар $(a, b)$ и $(c, d)$, таких, что $(a, b) \leqslant(c, d)$, из $\mathbf{P}_{\mathfrak{Z}}(a, b)=1$ следует $\mathbf{P}_{\mathfrak{I}}(c, d)=1$, а из $\mathbf{P}_{\mathfrak{Q}}(c, d)=0$ следует $\mathbf{P}_{\mathfrak{Q}}(a, b)=0$. Отсюда получаем, что предикат $\mathbf{P}_{\mathfrak{Z}}$ является монотонным относительно частичного порядка $\leqslant$, то есть что из $(a, b) \leqslant(c, d)$ следует $\mathbf{P}_{\mathfrak{Q}}(a, b) \leqslant \mathbf{P}_{\mathfrak{Q}}(c, d)$.

Пару $(a, b)$ назовем нижней единицей для $\mathbf{P}_{\mathfrak{L}}$, если $\mathbf{P}_{\mathfrak{L}}(a, b)=1$ и $\mathbf{P}_{\mathfrak{Q}}(c, d)=0$ для любого $(c, d)<(a, b)$. Пусть $\mathbf{U}\left[\mathbf{P}_{\mathfrak{L}}\right]-$ множество всех нижних единиц для $\mathbf{P}_{\mathfrak{L}}$. Ясно, что предикат $\mathbf{P}_{\mathfrak{L}}$ однозначно определяется указанием $\mathbf{U}\left[\mathbf{P}_{\mathfrak{Q}}\right]$. В случае, когда $\mathbf{U}\left[\mathbf{P}_{\mathfrak{L}}\right] \neq \varnothing$, назовем минимальной единицей для $\mathbf{P}_{\mathfrak{L}}$ ту из нижних единиц для $\mathbf{P}_{\mathfrak{L}}$, у которой первая координата равна числу $\min \left\{a \mid(a, b) \in \mathbf{U}\left[\mathbf{P}_{\mathbb{Q}}\right]\right\}$.

Пусть $\mathbf{U}\left[\mathbf{P}_{\mathfrak{l}}\right] \neq \varnothing$, и пусть $\left(a_{0}, b_{0}\right)$ - минимальная единица для $\mathbf{P}_{\mathfrak{l}}$. Легко убедиться, что $\mathbf{P}_{\mathfrak{Q}}(a, 0)=1$ для любого натурального числа $a \geqslant a_{0}+b_{0}$. Отсюда следует, что в случае, когда существует минимальная единица для $\mathbf{P}_{\mathfrak{L}}$, существует и максимальная единица для $\mathbf{P}_{\mathfrak{L},}$, то есть, есть нижняя единица для $\mathbf{P}_{\mathfrak{L},}$, у которой первая координата равна числу $a_{1}=\max \left\{a \mid(a, b) \in \mathbf{U}\left[\mathbf{P}_{\mathfrak{2}}\right]\right\}$. Легко показать, что максимальная единица имеет вид $\left(a_{1}, 0\right)$, и что для любого $a_{0} \leqslant x \leqslant a_{1}$ существует $y \in \mathbf{N}_{0}$ такой, что $(x, y) \in \mathbf{U}\left[\mathbf{P}_{\mathbb{R}}\right]$.

Определим также частичную функцию $f_{\mathbf{P}_{2}}: \mathbf{N} \rightarrow \mathbf{N}_{0}$ так, что

$$
f_{\mathbf{P}_{\mathfrak{L}}}(x)=\min \left\{y^{\prime} \mid \mathbf{P}_{\mathfrak{L}}\left(x, y^{\prime}\right)\right\}
$$

функция $f_{\mathbf{P}_{\mathfrak{I}}}$ является полностью неопределенной, если $\mathbf{U}\left[\mathbf{P}_{\mathfrak{Q}}\right]=\varnothing$. Назовем эту функцию характеристической функцией предиката $\mathbf{P}_{\mathfrak{L}}$.

Пусть $\mathbf{U}\left[\mathbf{P}_{\mathfrak{Q}}\right] \neq \varnothing$, и пусть $\left(a_{0}, b_{0}\right)$ и $\left(a_{1}, 0\right)$ - минимальная и максимальная единица для $\mathbf{P}_{\mathfrak{L}}$, соответственно. Ясно, что функция $f_{\mathbf{P}_{\mathfrak{I}}}$ определена для всех $x \geqslant a_{0}$, и что $f_{\mathbf{P}_{\mathfrak{Q}}}(x)=0$ для всех $x \geqslant a_{1}$. Поскольку из $d>0$ и $\mathbf{P}_{\mathfrak{L}}(c, d)=1$ следует, что $\mathbf{P}_{\mathfrak{Z}}(c+1, d-1)=1$, функция $f_{\mathbf{P}_{\mathfrak{Z}}}$ строго убывает на множестве $\mathbf{p}_{1}\left(\mathbf{U}\left[\mathbf{P}_{\mathfrak{Q}}\right]\right)$. Удобно представлять функщию $f_{\mathbf{P}_{\mathfrak{I}}}$ графическим образом. Свяжем прямым отрезком точку $(0,0)$ и $\left(a_{0}, b_{0}\right)$, а также пары точек вида $\left(n, f_{\mathbf{P}_{\mathfrak{l}}}(n)\right)$ и $\left(n+1, f_{\mathbf{P}_{\mathfrak{l}}}(n+1)\right)$ для любого $n \geqslant a_{0}$. Полученная ломаная имеет следующее свойство: она проходит через все нижние единицы, и для любой целочисленной точки $(x, y), x \geqslant a_{0}$, которая лежит на этой ломаной или над ней, значение предиката $\mathbf{P}_{\mathfrak{L}}$ равно 1 , для всех остальных (лежащих под этой ломаной или 


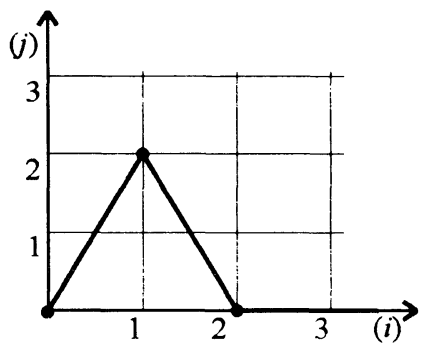

Рис. 1.

слева от прямой $x=a_{0}$ ) это значение равно 0; эту ломаную назовем характеристическим графиком предиката $\mathbf{P}_{\mathfrak{2}}$.

Наконец, сделаем одно замечание по поводу употребления термина предикат. Мы будем использовать его в самом общем смысле: под $n$-местным предикатом на множестве $M$ понимаем произвольную $n$-местную функцию, определенную на $M$ и принимающую значения 0 и 1 . Тогда под областью определения данного предиката подразумеваем множество всех наборов значений аргументов, на которых этот предикат принимает значение 1.

\section{3. Поведение коллектива автоматов в лабиринте}

Рассмотрим задачу синтеза для коллективов автоматов в лабиринтах. Сначала рассмотрим случай плоских мозаичных лабиринтов и выясним, какие минимальные по числу автоматов коллективы могут обходить все такие лабиринты.

Теорема 1. Для класса $\mathfrak{2}_{\mathrm{M}}^{\mathrm{K}}$ и предиката $\mathbf{P}_{\mathfrak{L}_{\mathrm{M}}}$ справедливо равенство

$$
\mathbf{U}\left[\mathbf{P}_{\mathfrak{L}_{M}}\right]=\{(1,2),(2,0)\},
$$

при этом некоторые коллективы типа $(1,2)$ обходят лабиринты из п клеток класса $\mathfrak{Q}_{\mathrm{M}}^{\mathrm{K}}$ за время $O\left(n^{3}\right)$, а типа $(2,0)-$ за время $O\left(n^{2}\right)$ и останавливаются после обхода.

Характеристический график предиката $\mathbf{P}_{\mathfrak{Q}}$ приведен на рис. 1. В [35] показано, что $\mathbf{P}_{\mathfrak{Q}}(1,5)=1$, причем сушествуют некоторые коллективы типа $(1,5)$, которые обходят любой лабиринт из $\mathfrak{Q}_{\mathrm{M}}^{\mathrm{K}}$ и останавливаются после обхода. В [4] дан эскиз доказательства того, что $\mathbf{P}_{\mathfrak{Q}_{\mathfrak{M}}^{\mathrm{k}}}(1,2)=\mathbf{P}_{\mathfrak{Q}_{\mathfrak{M}}^{\mathrm{K}}}(2,0)=1$ (полное доказательство можно найти, например, в [49]). Отметим, что на самом деле в упомянутых работах вместо класса $\mathfrak{2}_{\mathrm{M}}^{\mathrm{K}}$ рассматривался класс $\mathfrak{Q}_{ш}^{\kappa}$, но, как нетрудно заметить, достаточно внести небольшие изменения в доказательства соответствующих утверждений для класса $\mathfrak{Q}_{ш}^{\mathrm{k}}$ чтобы получить доказательства тех же самых утверждений для класса $2_{M}^{2}$. Приведенный в [4] алгоритм обхода оказался весьма плодотворным: он использовался в разных вариациях при решении некоторых других проблем. Опишем его здесь в основных чертах в случае класса $\mathfrak{2}_{\mathrm{w}}^{\mathrm{K}}$.

Пусть $L-$ некоторый конечный плоский шахматный лабиринт и $h-$ некоторая его (конечная или бесконечная) целочисленная дыра. Под границей $\mathrm{Nb}(h)$ дыры $h$ понимаем множество всех вершин лабиринта $L$, которые находятся на расстоянии, не превосходящем $\sqrt{2}$, от некоторой вершины дыры $h$. Обозначим через $\preccurlyeq$ порядок в $\mathbf{Z}^{2}$ такой, что 


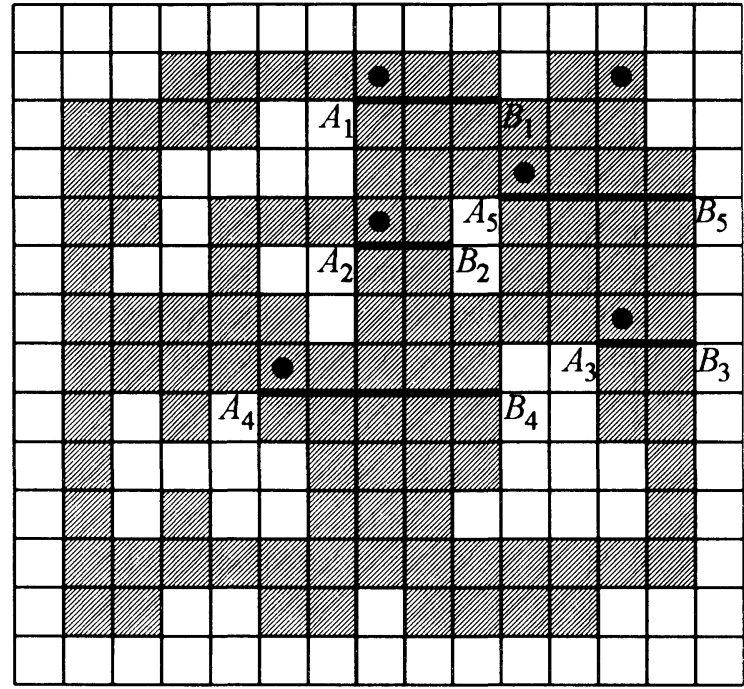

Рис. 2.

$(a, b) \preccurlyeq(c, d)$, где $(a, b),(c, d) \in \mathbf{Z}^{2}$, тогда и только тогда, когда $(b<d) \vee(b=d \wedge a \leqslant c)$. Поле $v_{0} \in \mathrm{Nb}(h)$ называется $h$-особенным полем, если $v \preccurlyeq v_{0}$ для любого $v \in \mathrm{Nb}(h)$. Ясно, что для любой дыры существует только одно такое поле. Поле лабиринта $L$ называется особенным, если оно $h$-особенно для некоторой его целочисленной дыры $h$. Легко убедиться, что если бы особенные поля всех конечных Z-дыр лабиринта $L$ были маркированы (скажем, символом •), то было бы легко построить автомат, который обходит таким способом размеченный лабиринт $L$. Для такой цели годится, например, немного измененный вариант автомата, обходяшего все конечные плоские шахматные лабиринты без конечных Z-дыр, упомянутый в [54] (он переходит на другую горизонталь только если слева от него находится поле какой-то Z-дыры); достаточно к программе этого автомата добавить следующие указания: если $u$ - маркированная вершина, то ходы с $u$ на $u \mathbf{s}$ и с $u \mathbf{s}$ на $u$ запрещаются (если автомат делает шаг такого типа, то он должен сразу вернуться на исходное поле, то есть, на поле, с которого совершил такой шаг). Если автомат следует таким указаниям, то он не переходит воображаемый горизонтальный отрезок с концами на границах двух соседних дыр, разделяющий горизонталь маркированного поля $u$ от горизонтали его южного соседа $u \mathbf{s}$ (все воображаемые отрезки, данного на рис. 2 лабиринта, суть отрезки $A_{i} B_{i}, i \in \overline{5}$ ). Таким образом, маркировка особенных полей способствует разрезанию лабиринта по горизонтальным отрезкам, что превращает его в односвязный лабиринт. Следовательно, задача обхода сводится к тому, чтобы построить автомат, который обнаруживает особенные поля. Оказывается, что для такой цели годится автомат с одним счетчиком. Этот автомат, оказавшись на поле $v$ границы некоторой дыры $h$, может (если точку $v$ вообще можно подозревать в особенности), поворачиваясь вокруг дыры надлежащим способом и возвращаясь в $v$, ответить как на вопрос, является ли поле $v$ особенным полем дыры $h$, так и на вопрос, является ли эта дыра бесконечной или конечной. Следовательно, он может узнать, маркировано это поле или нет (при этом в счетчике всегда хранится число, не превосходящее периметр дыры). Сочетая надлежащим способом алгоритм этого автомата и автомата, который мы описали выше, получаем, 
наконец, автомат со счетчиком, который обходит любой плоский конечный шахматный лабиринт. Этот автомат может фиксировать факт обхода, поскольку его повторное исследование на особенность особенного поля бесконечной дыры, фактически обозначает, что он уже обошел лабиринт. Легко смоделировать работу этого автомата коллективами автоматов типа $(1,2)$ и $(2,0)$, причем оценки времени обхода для них неявно следуют из данного алгоритма. Также неявно из [4] следует, что автомат со счетчиком обходит класс всех конечных плоских шахматных лабиринтов за время $O\left(n^{2}\right)$. Поскольку формальное отличие шахматных и мозаичных лабиринтов не сушественно в этих построениях, те же результаты имеют место и для класса $\mathfrak{Q}_{\mathrm{M}}^{\mathrm{K}}$, что утверждается в теореме 1 .

К сожалению, данный алгоритм можно применить только к мозаичным лабиринтам, поскольку автоматы, которые двигаются согласно этому алгоритму, не только учитывают, в каких направлениях выходят из текушей вершины ребра (сила компаса), но и сравнивают на равенство ординаты вершин границы (на границе они могут обнаружить все поля, которые находятся на одном и том же уровне). Поэтому эти алгоритмы невозможно применить к прямоугольным лабиринтам. В [13] удалось осуществить обобщение теоремы 1 на случай плоских прямоугольных лабиринтов. В ней использован алгоритм из [1], с помощью которого автомат, начиная свою работу с любой внутренней вершины (с вершины, которая не принадлежит границе бесконечной дыры) конечного плоского прямоугольного лабиринта, выходит на его внешнюю границу (границу его бесконечной дыры), причем этот алгоритм использует только силу компаса. Этот алгоритм в [13] расширен (выделены его основные ключевые моменты) и усовершенствован, и с его помощью теорема 1 обобщена на случай плоских прямоугольных лабиринтов с соответствующими оценками времени обхода вида $O\left(n^{4}\right)$ и $O\left(n^{3}\right)$, а для автомата со счетчиком и одним камнем это время есть $O\left(n^{2}\right)$. Неизвестно, обходит ли автомат со счетчиком все прямоугольные лабиринты.

Окончательный шаг, необходимый для полного утверждения справедливости теоремы 1 , был сделан в работах $[18,19]$. Сначала в [18] в эскизном виде, а потом подробно в [19] было дано доказательство того, что $\mathbf{P}_{\mathfrak{L}_{M}}(1,1)=0$. Важно отметить, что в отличие от случая одного автомата, для любого коллектива типа $(1,1)$ нельзя построить правильную ловушку.

Следует заметить, что, как и в случае класса $\mathfrak{2}_{\mathrm{M}}^{\mathrm{K}}(m), m \in \mathbf{N}$, из [54], возможно вложенное расслоение класса $\mathfrak{Q}_{\mathrm{M}}^{\mathrm{K}}$ такое, что для каждого слоя имеется автомат с одним камнем, обходящий его. Там это расслоение было по размерам дыр, а сейчас по количеству дыр.

Теорема 2. Для любого $k \in \mathbf{N}$ существует коллектив типа $(1,1)$, обходячий все конечные плоские (шахматные) мозаичные лабиринты, у которых не более $k$ (челочисленных) дыр, при этом автомат имеет не более $C^{k}$ состояний, где $C$ - постоянная.

В [37] было установлено, что существует коллектив типа $(1,1)$, который обходит все конечные плоские шахматные лабиринты (начиная обход с любой вершины любого такого лабиринта), имеющие не более двух Z-дыр (иными словами, поскольку в это число обязательно входит единственная бесконечная дыра, то имеющие не более одной конечной Z-дыры). В [38] это утверждение доказано в случае, когда у лабиринта не больше трех Z-дыр. Затем в [25] была доказана первая часть теоремы 2 для случая конечных плоских шахматных лабиринтов, а позже в [12] была установлена оценка для числа состояний автомата и упрощено доказательство первой части теоремы. Приведем здесь схему доказательства этой теоремы из [12].

Исследование начинается с плоских $R$-графов с ограничением $d \geqslant 2$ на степень вершин (см. [54]), у которых вращение в любой вершине соответствует вращению, которое, 
естественно, задается положительной ориентацией в плоскости. Обозначим этот класс плоских графов через $\mathscr{S}_{R}^{+}(d)$. В лабирингах этого класса рассматриваются $R$-автоматы, у которых кроме одного обязательного камня (служащего для его ориентации, поскольку у него нет возможности пользоваться компасом) есть еще один реберный камень; чтобы как-то отличать их, первый камень назовем связанным, а второй - свободным камнем или просто камнем. Обход лабиринтов из $\mathscr{S}_{R}^{+}(d)$ такими автоматами понимается в немного измененном виде: автомат обходит лабиринт, если за некоторое конечное время все ребра лабиринта хотя бы один раз бывают маркированными его свободным камнем. Вершина лабиринта $L \in \mathcal{B S}_{R}^{+}(d)$ является вершиной ранга $m$, если она лежит на границе точно $m$ различных граней лабиринта. Назовем вилочной вершину, у которой ранг больше или равен 3. Обозначим через $\left(S_{R}^{+}(d, k)\right.$ класс всех лабиринтов из $\left(S_{R}^{+}(d)\right.$, у которых не более $k$ вилочных вершин. В [12] сначала показано, что для любого $k$ сушествует универсальный обходчик данного выше тила для класса $\left(s_{R}^{+}(3, k)\right.$ с числом состояний, не превосходящим $C^{k}$ (понятно, что в случае этого класса ранг всех вилочных вершин равен трем). При этом, если вообще есть вилочные вершины, то этот автомат обязательно останавливается. Потом это утверждение обобщается на случай любого $d \geqslant 3$.

Условие, которое накладывалось на количество вершин с рангом не менее 3, заменяется далее условием, требующим чтобы в $R$-графе класса $\left(\oiint_{R}^{+}(d)\right.$ было не больше $k$ граней. Но как легко заметить, в $R$-графе, удовлетворяющем этому условию, не больше $2 k$ вершин с рангом не менее 3 , то есть такие лабиринты являются элементами множества $\left(\mathcal{S}_{R}^{+}(d, 2 k)\right.$. Но тогда и для них можно построить универсальный обходчик, у которого не более $\left(C_{1}\right)^{k}=C^{2 k}$ состояний.

Наконец, если дан конечный плоский шахматный лабиринт (случай мозаичного лабиринта можно считать более легким), у которого не более $k$ целочисленных дыр, то поступаем следующим образом. В качестве опорного алгоритма используем алгоритм обхода автомата, являющегося универсальным обходчиком для класса всех конечных шахматных лабиринтов без Z-дыр, и таким образом избавимся от всех мелких дыр (если на лабиринт смотреть как на мозаичный). В полученном (виртуальном) мозаичном лабиринте остается именно $k$ дыр (не целочисленных). Это значит, что автомат, который мы строим, считает, что каждый шахматный лабиринт указанного типа принадлежит классу $(\$)_{R}^{+}(4,2 k)$. Опорный алгоритм теперь легко сочетать с алгоритмом автомата, который решает задачу в предыдущем случае, используя вместо реберного камня один вершинный, поскольку в мозаичном случае автомат может ориентироваться в любой вершине по четырем основным направлениям (у него есть компас).

Возможности коллективов автоматов при обходе лабиринтов много шире, чем возможности независимых систем автоматов. Об этом свидетельствуют следующие утверждения, в которых речь идет о конечных и бесконечных плоских мозаичных лабиринтах.

Теорема 3 ([23, 51]). Для класса $\mathfrak{\complement}_{\mathrm{M}}$ и предиката $\mathbf{P}_{\mathfrak{L}_{\mathrm{M}}}$ имеют место отночения

$$
\{(2,3),(3,2),(4,1),(5,0)\} \subseteq \mathbf{U}\left(\mathbf{P}_{\mathfrak{L}_{M}}\right), \quad \mathbf{P}_{\mathfrak{L}_{M}}(1,3)=0, \quad \mathbf{P}_{\mathfrak{U}_{M}}(1,5)=1 .
$$

В [3] установлено, что $\mathbf{P}_{\mathfrak{L}_{\mathrm{M}}}(1,7)=1$. Впоследствии доказательство этого факта из [3] уточнено в [10]. Дальнейшее развитие идей из [3] удалось осуществить в [36], где с помощью алгоритма обхода конечных шахматных лабиринтов (см. [4]), показано, что $\mathbf{P}_{\mathfrak{Q}_{M}}(1,5)=1$. Наконец, с помощью достаточно общей конструкции теорема 3 была доказана в $[23,51]$. Ее доказательство проводилось посредством конструирования соответствующей ловушки для коллективов автоматов типов $(1,3),(2,2),(3,1)$ и $(4,0)$. Затем строились примеры коллективов типов $(2,3),(3,2),(4,1)$ и $(5,0)$, которые обходят все 


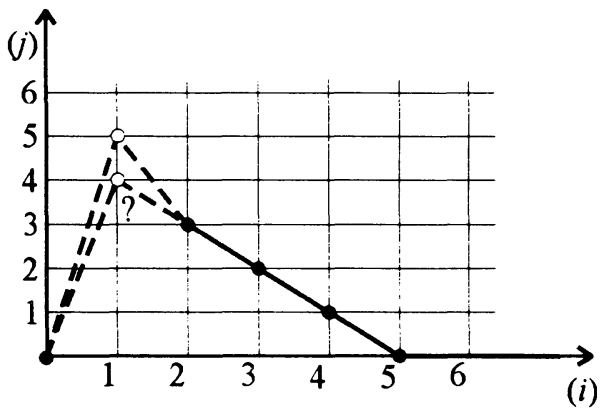

Рис. 3.

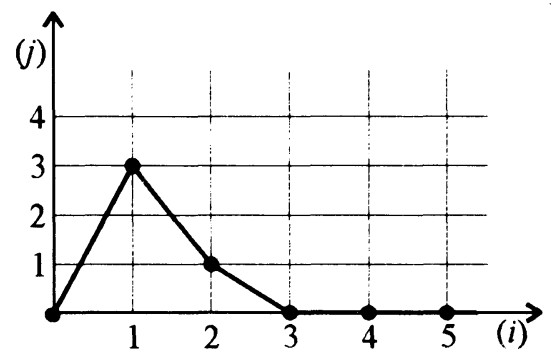

Рис. 4.

плоские мозаичные лабиринты. Все возможные варианты для характеристического графика предиката $\mathbf{P}_{\mathfrak{L}_{M}}$ приведены на рис. 3. Знаком? отмечена точка $(1,4)$, в которой вопрос о значении предиката $\mathbf{P}_{\mathfrak{L}_{\mathrm{M}}}$ открыт.

Так же, как и для независимых систем автоматов, интересно выяснить, какие достаточно широкие классы лабиринтов могут быть обойдены коллективами простых типов. Заметим, что если перейти к классу $\mathfrak{2}_{M}^{\prime}$ всех плоских мозаичных лабиринтов, не содержащих бесконечных дыр, то остается справедливым утверждение, аналогичное теореме 3.

Теорема 4 ([23, 51]). Для класса $\mathfrak{Z}_{\mathrm{M}}^{\prime}$ и предиката $\mathbf{P}_{\mathfrak{L}_{\mathrm{M}}^{\prime}}$ имеет место отношение

$$
\{(2,3),(3,2),(4,1),(5,0)\} \subseteq \mathbf{U}\left[\mathbf{P}_{\mathcal{L}_{M}^{\prime}}\right], \quad \mathbf{P}_{\mathcal{L}_{M}^{\prime}}(1,3)=0, \quad \mathbf{P}_{\mathcal{L}_{M}^{\prime}}(1,5)=1
$$

Отсюда следует, что $\mathbf{U}\left[\mathbf{P}_{\mathcal{L}_{M}}\right]$, а также $\mathbf{U}\left[\mathbf{P}_{\mathfrak{L}_{M}^{\prime \prime}}\right]$, есть или $\{(1,5),(2,3),(3,2),(4,1),(5,0)\}$, или $\{(1,4),(2,3),(3,2),(4,1),(5,0)\}$. Существует гипотеза, что

$$
\mathbf{U}\left[\mathbf{P}_{\mathfrak{L}_{M}}\right]=\mathbf{U}\left[\mathbf{P}_{\mathfrak{L}_{M}^{\prime \prime}}\right]=\{(1,5),(2,3),(3,2),(4,1),(5,0)\}
$$

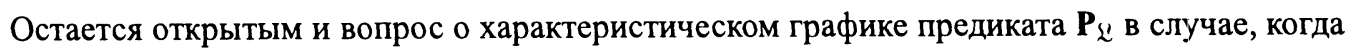
I - класс всех плоских шахматных лабиринтов, имеющих конечное число конечных целочисленных дыр, как и во всех случаях подобных этому (вместо шахматных можно рассматривать мозаичные лабиринты, вместо конечных дыр бесконечные, и т. п.).

В случае, когда класс \& состоит только из лабиринта $\mathbf{Z}^{2}$, можно показать, что характеристический график предиката $\mathbf{P}_{\mathfrak{L}}$ имеет вид, указанный на рис. 4 . Это следует из 
результатов статьи [44], где показано, что существуют коллективы типов $(1,3)$ и $(2,1)$, которые обходят плоскость $\left(\mathbf{Z}^{2}\right)$, но не существуют коллективы типов $(1,2)$ и $(2,0)$, которые делают то же самое.

Интересно отметить, что уже для плоского мозаичного древовидного лабиринта $L$ с двумя бесконечными ветвями $P_{L}(1,1)=0$ (см. ниже), а также, что для полуплоскости $\overline{\mathbf{Z}^{2}}$ справедливо равенство $\mathbf{P}_{\overline{\mathbf{Z}^{2}}}(1,1)=1$, но $\mathbf{P}_{\overline{\mathbf{Z}^{2}}}(1,0)=0$ (см. [37]).

В качестве некоторых специальных классов мозаичных (шахматных) лабиринтов рассматривались и (целочисленные) одномерные ленты и полуленты. Под одномерной лентой понимается подлабиринт целочисленной плоскости $\mathbf{Z}^{2}$, порождаемый точками $\{(x, 0) \mid x \in \mathbf{Z}\}$; под полулентой с началом в точке $n_{0} \in \mathbf{Z}$ понимается подлабиринт лабиринта $\mathbf{Z}^{2}$, определенный или множеством

$$
R_{n_{0}}=\left\{(n, 0) \in \mathbf{Z}^{2} \mid n \geqslant n_{0}\right\}
$$

(правая лента с началом в $n_{0}$ ), или множеством

$$
L_{n_{0}}=\left\{(n, 0) \in \mathbf{Z}^{2} \mid n \leqslant n_{0}\right\}
$$

(левая лента с началом в $n_{0}$ ). В [44] показано, что автомат с одним камнем не может обойти ни одномерную ленту, ни все одномерные полуленты, тогда как конечный автомат с двумя камнями может это сделать. Если позволить размечать вершины лент (полулент) символами любого конечного алфавита, то получаются помеченные ленты (полуленты). В случае помеченных лент там же показано, что для любого коллектива типа $(1,1)$ любая помеченная лента (любым способом размеченная лента) является его ловушкой.

Перейдем к рассмотрению лабиринтов более общего вида. Имеет место следующее угверждение.

Теорема 5. Не существует коллектива автоматов, обходящего все 3-мерные инициальные конечные мозаичные лабиринты.

Доказательство этой теоремы проводится посредством построения ловушки для заданного коллектива автоматов. Это утверждение было впервые сформулировано и частично обосновано в [3]. Его доказательство дается в [15], где показано, что для любого конечного множества коллективов автоматов существует конечная 3-мерная мозаичная инициальная ловушка ограниченной толщины - все ее вершины лежат только в двух плоскостях.

Назовем бесконечный мозаичный $n$-мерный лабиринт $L$ мозаичной $n$-мерной универсальной ловушкой, если любой коллектив автоматов в $\mathbf{R}^{n}$, стартуя из любого набора вершин лабиринта $L$, не обходит его. Показано (см. [52]), что так же, как и в случае независимых систем автоматов в плоскости, существует мозаичная $n$-мерная универсальная ловушка для любого $n \geqslant 3$ (ясно, что при этом достаточно было показать, что такая ловушка существует для $n=3$ ).

Назовем мозаичную $n$-мерную универсальную ловушку однородной, если для любого коллектива $\mathscr{A}$ автоматов существует $r=r(\mathscr{A})$ такое, что при любых $v_{1}, \ldots, v_{n}$ из $V(L)$ справедливо включение

$$
\operatorname{Int}\left(\mathscr{A}, L_{v_{1}, \ldots, v_{n}}\right) \subseteq \mathrm{Bl}_{n}\left(v, r+\operatorname{diam}\left\{v_{1}, \ldots, v_{n}\right\}\right)
$$

для некоторого $v \in \mathbf{Z}^{n}$.

Теорема 6 ([52]). Существуют 3-мерные однородные ловушки. 
Из теоремы 3 следует, что для коллектива автоматов в классе всех плоских мозаичных лабиринтов в общем случае ловушки не существует. С другой стороны, в 3-мерном случае для любого коллектива автоматов можно найти ловушку даже среди всех конечных 3-мерных мозаичных лабиринтов (см. теорему 5). В связи с этим возникает вопрос, можно ли, не выходя за пределы некоторого класса планарных лабиринтов, строить ловушки (конечные или бесконечные) для произвольных коллективов автоматов. Ответ на последний вопрос является положительным. При этом оказывается, что можно ограничиться планарными графами (без петель и кратных ребер), размеченными по ребрам, то есть, имеющими в наличии систему направлений, причем эта система дана в виде раскраски ребер графов, а именно, в виде раскраски дуг соответствующих симметрических орграфов, причем цвета (направления) дуг, принадлежащих одному и тому же ребру, одинаковые. Оказывается, что для построения планарной ловушки такого типа для произвольного коллектива достаточно ограничиться тремя красками.

Сделаем некоторые замечания. Допустим, что краски, которыми раскрашиваются ребра планарных графов, упорядочены некоторым способом. Тогда любая реберная раскраска данного планарного графа задает определенную систему вращения этого графа, и это вращение необязательно является совпадающим с вращением, определенным некоторой его плоской укладкой (с помощью положительной ориентации на плоскости). Также заметим, что если при раскраске ребер использовалось $k$ красок, то есть если данный графф является $k$-цветным по ребрам, то он является графом с ограничением $k$ на степень вершин (инцидентные ребра должны быть окрашены в различные цвета). Имеет место следующая теорема.

Теорема 7 ([32]). Для любого коллектива автоматов существует планарная 3-цветная по ребрам ловушка.

Аналогичный вопрос может быть поставлен по отношению к плоским графам, например, таким, которые принадлежат множеству $\mho_{R}^{+}(3)$. При этом ограничимся регулярными плоскими графами степени 3 (у которых все вершины степени 3); такие графы называются плоскими кубическими графами. Исследовалось поведение коллективов $R$-автоматов в таких графах. Автоматы не имели вершинных камней, но их, в принципе, можно снабдить ими, только при этом на эти камни уже нельзя смотреть как на самые простые $R$-автоматы (у $R$-автоматов есть реберные камни). Установлено, что коллектив $R$-автоматов типа $(4,0)$ не может обойти все плоские кубические лабиринты [26]. Ранее в [4] это было установлено для случая $(3,0)$. Вопрос о существовании коллектива $R$-автоматов, который обходит любой плоский кубический граф, остается открытым. Сушествует гипотеза, что такого коллектива не существует.

Как мы уже отмечали, в [12] было установлено, что для класса $\mathcal{S}_{R}^{+}(d, k)$, а также для класса всех графов из $\left(S_{R}^{+}(d)\right.$, у которых не больше $k$ граней, существует универсальный $R$-автомат с одним (дополнительным) реберным камнем, у которого не более, чем $e^{O(k)}$ состояний.

В $[16,17]$ исследуются возможности $R$-автоматов, имеющих (свободные) реберные или вершинные камни, при обходе лабиринтов класса $\mathscr{S}_{R}(d)$. В [16] показано, что можно построить $R$-автомат с $O(n)$ реберными камнями, который обходит любой $n$-вершинный лабиринт класса $\left(_{R}(d)\right.$ за время $O(n)$ и останавливается после обхода. В [17] показано, что существует $R$-автомат с $O(n)$ вершинными камнями, который обходит любой $n$-вершинный лабиринт класса $\left(S_{R}(d)\right.$ за время $O\left(n^{2}\right)$.

Одним из примеров класса специальных лабиринтов является лабиринт, условно называемый сигнатурным. Он представляет собой плоский мозаичный лабиринт, содержащий 


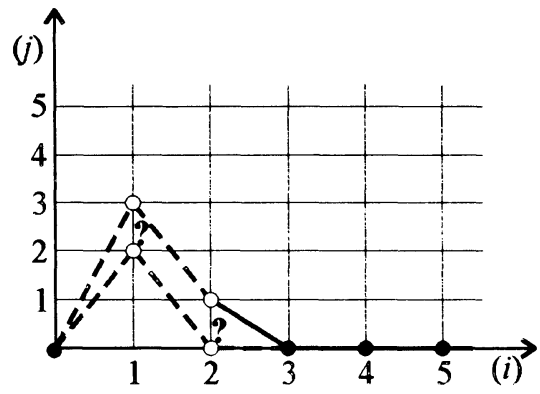

Рис. 5.

начало координат, точку $(0,0)$, в котором каждой вершине приписан набор знаков координат этой вершины, то есть каждой точке $(x, y) \in \mathbf{Z}^{2}$ приписана метка вида $(\operatorname{sgn} x, \operatorname{sgn} y)$ (в таком случае считаем, что точке $(0,0)$ приписана метка $(0,0))$. В [67] рассмотрена задача поиска коллективом автоматов точки $(0,0)$ в сигнатурном лабиринте; при этом для автоматов этого коллектива функщии переходов и выходов уточняются с учетом указанных меток вершин. На множестве пар $(i, j) \in \mathbf{N}_{0} \times \mathbf{N}$ вводим предикат $\mathbf{P}$, характеризующий наличие или отсутствие коллектива автоматов типа $(i, j)$, находящего начало координат. Этот предикат, очевидно, является монотонным. Аналогичным образом, как и в случае предиката обхода, можно рассматривать множество его нижних единиц и определить соответствующий характеристический график.

Теорема 8. Для предиката $\mathbf{P}$ поиска начала координат в сигнатурном лабиринте имеют место равенства

$$
\mathbf{P}(1,1)=0, \quad \mathbf{P}(1,3)=1, \quad \mathbf{P}(2,1)=1, \quad \mathbf{P}(3,0)=1 .
$$

$\mathrm{B}$ [67] показано, что $\mathbf{P}(1,0)=0$ и $\mathbf{P}(4,0)=1$. Первое из этих двух равенств является прямым следствием того, что для любого автомата сушествует правильная ловушка (такую ловушку можно, например, поместить в первый квадрант, и мозаичным змеевидным лабиринтом связать выход этой ловушки с началом координат). Но это же равенство является следствием и более сильного утверждения, состояшего в том, что $\mathbf{P}(1,1)=0$, причем его можно доказать, применяя практически тот же способ, который использовался в [67] для случая одного автомата. Это делается следующим способом. Пусть $\mathscr{A}-$ некоторый коллектив типа $(1,1)$. Как мы уже видели, для любого коллектива автоматов типа $(1,1)$ любая одномерная, помеченная любым способом лента является ловушкой. Возьмем сигнатурную одномерную ленту, которая не проходит через точку $(0,0)$, и запустим этот коллектив. Поскольку он не обходит ее, сущєствует клетка ленты, которую коллектив $\mathscr{A}$ не посещает. Связав эту точку мозаичной линией (мозаичным змеевидным лабиринтом) с вершиной $(0,0)$, мы получим, отмечая надлежащим образом все вершины этого лабиринта, сигнатурный лабиринт, в котором коллектив $\mathscr{A}$ не решает задачу поиска начала координат. На рис. 5 в области, ограниченной пунктирной линией, вид характеристического графика предиката Р неизвестен (знаком ? отмечены те точки, в которых значение предиката $\mathbf{P}$ неизвестно).

Особым направлением исследований является выявление возможности анализа лабиринтов с помощью различных обобщений автоматов таких, как автоматы со счетчиками, магазинами, стеками, многоголовочные и др. 
В [7] установлено, что не существует автомата с магазином, который обходит все конечные кубические плоские графы, в то время как линейно ограниченная машина Тьюринга эту задачу решает. Там же отмечается, что сушествует автомат с магазином, который может обойти все плоские конечные мозаичные лабиринты.

В [6] показано, что если допустить существование одного магазина у автомата, то можно легко построить автомат, который обходит за время $O(n)$ ( $n-$ число вершин) любое конечное $R$-дерево $L$ с ограничением $d \geqslant 2$ на степень вершин и останавливается после обхода (отмечается, что один автомат и один камень также решают эту задачу). Прямым следствием этого факта является возможность построения такого автомата для конечных плоских мозаичных древовидных лабиринтов, а тем самым и для конечных плоских односвязных шахматных лабиринтов. Эти результаты, по существу, являются положительным решением проблемы остановки для приведенных классов лабиринтов. Заметим, что эта проблема для тех же классов в случае автомата без магазина имеет отрицательное решение [6].

Проблема остановки для класса всех конечных $R$-деревьев с ограничением $d \geqslant 3$ остается нерешенной в случае автомата с одним счетчиком. Единственное, что удалось показать в [6] в связи с этим случаем, это то, что если сузить этот класс до класса всех центрированных $R$-деревьев с ограничением $d \geqslant 2$ на степень вершин $d$, то останавливающийся автомат такого типа уже существует (дерево является центрированным, если существует вершина, которая находится на одинаковом расстоянии от всех его концевых вершин).

В [14] введен такой тип конечной трехмерной мозаичной ловушки, которая может быть построена для каждого коллектива автоматов, однако существует двухголовочный автомат, для которого нельзя построить ловушку такого типа. Ловушки, которые строились, были инициальными ловушками для всех коллективов из $k$ автоматов, у которых не больше $l$ состояний, где $k$ и $l$ - любые заранее фиксированные натуральные числа. Здесь под многоголовочным ( $k$-головочным) автоматом понимается коллектив (содержащий $k$ ) автоматов со связью следующего вида: каждый автомат знает состояние любого другого автомата этого коллектива на любом шаге его работы. Это показывает, что при анализе лабиринтов возможности многоголовочных автоматов превышают возможности коллективов автоматов. Остается открытым вопрос, существуют ли соответствующие универсальные обходчики типа многоголовочного автомата для всех трехмерных (конечных) мозаичных лабиринтов и для всех плоских кубических графов.

В [44] показано, что автомат с одним магазином не обходит ни одномерную ленту, ни все одномерные полуленты, тогда как (конечный) автомат с одним камнем и одним счетчиком, автомат с двумя счетчиками или автомат с одним стеком могут обойти ленту. Выше было отмечено, что в случае помеченных лент для любого коллектива типа $(1,1)$ любая помеченная лента является его ловушкой. В противоположность этому, для автомата с одним счетчиком ленту всегда можно разметить так, что он уже обходит ее. При этом оказывается, что для подходящей разметки хватает символов 0 и 1. В [44] также показано, что существуют автомат с одним стеком или с двумя счетчиками; коллектив, состоящий из одного автомата со счетчиком и двух камней; коллектив, состоящий из одного конечного автомата и одного автомата со счетчиком; которые обходят плоскость, и что не существует коллектива, состоящего из автомата с одним магазином и одного камня, который обходит плоскость.

Хотя вышеизложенные результаты, относящиеся к проблеме обхода лабиринтов, касались только детерминированных коллективов автоматов, эту проблему можно было бы также исследовать и применительно к недетерминированным и даже вероятностным сис- 
темам автоматов. Проблема обхода одного класса лабиринтов вероятностными автоматами рассмотрена в [44]. Она решалась в контексте подтверждения факта преимущества вероятностных автоматов перед детерминированными при обходе лабиринтов. Вероятностные автоматы, рассматриваемые в [44], отличаются от обыкновенных конечных автоматов только тем, что они дополнительно снабжены еще и дискретными датчиками случайных чисел, которые по их запросу с вероятностью $1 / 2$ выдают или число 0 , или число 1 , и это число также является частью локальной информации, на основании которой автомат принимает решения, касаюшиеся своей дальнейшей работы. Иными словами, у этих автоматов есть добавочный вход, и на этом входе, на любом шаге их работы, стоит с вероятностью $1 / 2$ или число 0, или число 1. Обход лабиринта автоматами такого типа понимался в следуюшем смысле: вероятностный автомат обходит некоторый лабиринт, если с вероятностью 1 рано или поздно (а поэтому и бесконечное число раз) окажется в любой его вершине. Обход лабиринтов этими автоматами изучался по отношению к лабиринтам, которые представляли собою всевозможные размеченные по дугам симметрические орграфы, соответствующий граф которых является бесконечным плоским регулярным деревом степени 3. Одна точка таких лабиринтов выделена и обозначается через $O$. Система направлений состоит из символов 0,1 и 2 . Единственным ограничением на разметку является условие, что в любой вершине та дуга, по которой из этой вершины идет единственный простой путь в вершину $O$, всегда отмечена символом 0 (напомним, что под простым путем понимаем путь или ориентированный маршрут, у которого все вершины различны). В [44] показано, что не сушествует коллектива типа $(1,1)$, который обходит хотя бы один лабиринт данного типа, но, с другой стороны, существует конечный вероятностный автомат данного выше типа, который с вероятностью 1 побывает в каждой вершине любого графа данного вида.

В этой же работе также отмечено, что вероятностным автоматом можно смоделировать симметричное случайное блуждание точки в $\mathbf{Z}^{2}$, то есть на блуждающую точку можно смотреть как на вероятностный автомат простейшего типа (в случае вероятностных автоматов данного выше типа для соответствующего моделирования достаточно двух состояний). Поскольку при симметричном случайном блуждании на плоскости точка с вероятностью 1 попадает в любую вершину лабиринта $\mathbf{Z}^{2}$ (см., например, [71]), а любая система из двух автоматов, как и любая система из одного автомата и двух камней, не обходит плоскость, вероятностные автоматы оказываются в каком-то смысле сильнее коллективов автоматов типа $(1,2)$ и $(2,0)$.

Отметим, что таким же способом можно показать, что вероятностные автоматы сильнее коллективов автоматов типа $(1,3),(2,2),(3,1)$ и $(4,0)$, поскольку они обходят любой плоский мозаичный лабиринт (с вероятностью 1 попадают в любое его поле), а ни один коллектив указанного типа не является универсальным обходчиком для класса $\mathfrak{L}_{\mathrm{M}}$ (см. теорему 3).

Интересно также отметить, что при симметрическом случайном блуждании в $\mathbf{Z}^{3}$ (по шести направлениям) точка уже только с вероятностью $\approx 0,35$ попадает в любую его целочисленную вершину (см. [71]) и что до сих пор актуальна проблема нахождения алгоритма перемещения точки в пространстве, при котором она попадает с вероятностью 1 в любое поле пространства $\mathbf{Z}^{3}$. На тот же самый факт указывается и в [44], но при этом упоминается случай симметрического случайного блуждания точки в $\mathbf{Z}^{3}$ по восьми направлениям, что, по нашему мнению, в меньшей мере соответствует проблеме обхода мозаичных лабиринтов (хотя легче моделируется с помощью вышеуказанных случайных автоматов), и указывается, что соответствующая вероятность равна примерно 0,237 (более точной оценкой этой вероятности является число 0,239). Ясно, что если бы удалось 
найти вероятностный автомат, который обходит трехмерное пространство, то эта задача имела бы положительный ответ. Даже в случае, если такого автомата не существует, интересно было бы найти хотя бы коллектив вероятностных автоматов, минимальный по числу автоматов, который уже решает эту проблему. Отметим, что существует коллектив детерминированных конечных автоматов, например, типа $(2,3)$, который обходит $\mathbf{Z}^{3}$.

С проблемой обхода тесно связана и так называемая проблема встречи.

Пусть $\mathfrak{L}$ - класс лабиринтов и $\mathfrak{C}-$ некоторый класс коллективов автоматов, допустимых для $\mathfrak{2}$. Введем двухместный предикат М на множестве 5 следующим способом: для любых $\mathscr{A}_{1}, \mathscr{A}_{2} \in \mathfrak{C}$ положим $\mathbf{M}\left(\mathscr{A}_{1}, \mathscr{A}_{2}\right)=1$, если коллективы $\mathscr{A}_{1}$ и $\mathscr{A}_{2}$, помещенные в любые вершины любого лабиринта из $\mathfrak{L}$, встречаются, в противном случае положим

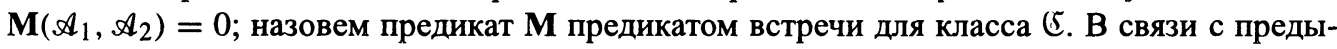
дущим определением сделаем следующие разъяснения. Во-первых, все автоматы рассматриваемых коллективов автоматов, здесь и в последующем, будем помещать в одну и ту же вершину (произвольную, но для всех одинаковую) данного лабиринта, то есть имеется в виду случай синхронизированных коллективов автоматов. Во-вторых, два коллектива, в самом общем смысле, встречаются, если в какой-то момент в некоторой вершине лабиринта окажутся представители обоих коллективов. Задача нахождения значения М по

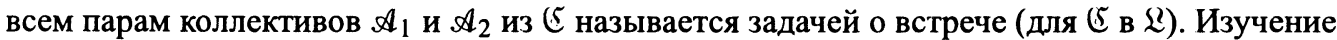
этого предиката ведется при разных допушениях. Приведем некоторые из них.

Пусть задан класс лабиринтов $\mathfrak{i}$ и два типа коллективов автоматов $\left(i_{1}, j_{1}\right)$ и $\left(i_{2}, j_{2}\right)$. Задача о типовой встрече для них состоит в выяснении, существуют ли коллективы автоматов указанных типов, которые при помещении каждого из них в произвольные стартовые вершины любого лабиринта указанного класса через некоторое время окажутся в ситуации, когда в некоторой вершине будут находиться представители каждого из коллективов, которые не являются камнями; эту задачу мы назовем $\left(i_{1}, j_{1} ; i_{2}, j_{2}\right)$-задачей о типовой встрече в $\mathfrak{L}$, а при $i_{1}=i_{2}$ и $j_{1}=j_{2}-\left(i_{1}, j_{1}\right)$-задачей о типовой встрече в $\mathfrak{Q}$ (при решении этих задач класс коллективов состоит из всех коллективов автоматов, допустимых для $\mathfrak{Q})$. В случае, когда речь идет о $(i, j)$-задаче и при этом требуется, чтобы коллективы, которые должны встречаться при любом стартовом положении в любом лабиринте из $\mathfrak{Q}$, совпадали (были двумя копиями одной и той же системы), говорим о сильной $(i, j)$-задаче о типовой встрече в 2 . При этом само собою разумеется, что в случае сильной $(i, j)$ задачи о типовой встрече, если автоматы первого коллектива встречают камни второго коллектива, то они их не отличают от своих. Правда, к программе коллектива, который служит оригиналом для обеих копий, должны быть добавлены указания о том, что делать, если в одной вершине окажется больше камней, чем положено (больше, чем их у этого коллектива имеется).

Пусть $\mathfrak{L}$ - некоторый класс лабиринтов. На множестве $\mathbf{N} \times \mathbf{N}_{\mathbf{0}} \times \mathbf{N} \times \mathbf{N}_{\mathbf{0}}$ определим предикат $\mathbf{M}_{\mathfrak{L}}^{0}$ следующим способом: $\mathbf{M}_{\mathfrak{L}}^{0}\left(i_{1}, j_{1}, i_{2}, j_{2}\right)=1$, если имеется положительное решение соответствующей $\left(i_{1}, j_{1} ; i_{2}, j_{2}\right)$-задачи о типовой встрече, и $\mathbf{M}_{\mathfrak{Q}}^{0}\left(i_{1}, j_{1}, i_{2}, j_{2}\right)=0$ в противном случае. Для $(i, j)$-задачи и сильной $(i, j)$-задачи о типовой встрече в 2 аналогичным образом определим, соответственно, предикаты $\mathbf{M}_{\mathfrak{L}}^{1}$ и $\mathbf{M}_{\mathfrak{L}}^{2}$ на множестве $\mathbf{N} \times \mathbf{N}_{0}$. Определим порядок $\leqslant$ на множестве $\bigcup_{k=1}^{\infty} \mathbf{N}_{0}^{k}$ следующим способом: соотношение $\left(a_{1}, \ldots, a_{m}\right) \leqslant\left(b_{1}, \ldots, b_{n}\right)$ имеет место тогда и только тогда, когда $m=n$ и $a_{i} \leqslant b_{i}$ для любого $1 \leqslant i \leqslant m$. Обозначим для любого $X \subseteq \bigcup_{k=1}^{\infty} \mathbf{N}_{0}^{k}$ через тот же самый символ $\leqslant$ порядок, индуцированный в $X$ определенным выше порядком. Ясно, что предикаты $\mathbf{M}_{\mathfrak{R}}^{0}$, $\mathbf{M}_{\mathfrak{L}}^{1}$ и $\mathbf{M}_{\mathfrak{L}}^{2}$ являются монотонными по отношению к порядку $\leqslant$, и поэтому их описание сводится к указанию нижних единиц, множество которых обозначаем, соответственно, 
через $\mathbf{U}\left[\mathbf{M}_{\mathfrak{L}}^{0}\right], \mathbf{U}\left[\mathbf{M}_{\mathfrak{L}}^{1}\right]$ и $\mathbf{U}\left[\mathbf{M}_{\mathfrak{L}}^{2}\right]$; эти множества определяют соответствуюшие предикаты так, что во всех точках, которые больше или равны некоторой нижней единице, значение предиката равно 1, а во всех остальных точках его значение равно 0. Для введенных предикатов очевидными, вытекающими прямо из определения, являются следующие тождества и неравенства:

$$
\begin{gathered}
\mathbf{M}_{\mathfrak{I}}^{0}\left(x, y, x_{1}, y_{1}\right)=\mathbf{M}_{\mathfrak{L}}^{0}\left(x_{1}, y_{1}, x, y\right), \quad \mathbf{M}_{\mathfrak{L}}^{1}(x, y)=\mathbf{M}_{\mathfrak{L}}^{0}(x, y, x, y) \\
\mathbf{M}_{\mathfrak{L}}^{2}(x, y) \leqslant \mathbf{M}_{\mathfrak{L}}^{1}(x, y) \leqslant \mathbf{M}_{\mathfrak{L}}^{0}\left(x_{2}, y_{2}, x_{3}, y_{3}\right),
\end{gathered}
$$

имеющие место для любых $x, x_{1} \in \mathbf{N}$ и $y, y_{1} \in \mathbf{N}_{0}$ и любых $x_{2}, x_{3} \in \mathbf{N}$ и $y_{2}, y_{3} \in \mathbf{N}_{0}$ таких, что $(x, y) \leqslant\left(x_{2}, y_{2}\right)$ и $(x, y) \leqslant\left(x_{3}, y_{3}\right)$.

Для некоторых классов $\mathfrak{Z}$ предикаты $\mathbf{M}_{\mathfrak{L}}^{0}, \mathbf{M}_{\mathfrak{L}}^{1}$ и $\mathbf{M}_{\mathfrak{L}}^{2}$ легко поддаются описанию. Например, в случае, когда $\mathfrak{Z}$ является классом всех трехмерных конечных мозаичных лабиринтов,

$$
\mathbf{U}\left[\mathbf{M}_{\mathfrak{l}}^{0}\right]=\mathbf{U}\left[\mathbf{M}_{\mathfrak{L}}^{1}\right]=\mathbf{U}\left[\mathbf{M}_{\mathfrak{L}}^{2}\right]=\varnothing,
$$

то есть $\mathbf{M}_{\mathfrak{I}}^{0}=\mathbf{M}_{\mathfrak{L}}^{1}=\mathbf{M}_{\mathfrak{L}}^{2}=0$ (этот результат доказывается с помощью более точного подбора ловушки из теоремы 5). Такой же ответ получается и в случае, когда множество $\mathfrak{L}$ содержит только одну 3 -мерную однородную ловушку. Для классов $\mathfrak{L}_{\mathrm{M}}^{\mathrm{K}}, \mathfrak{L}_{\mathrm{M}}^{6}$ и $\mathfrak{L}_{\mathrm{M}}$ задачу о встрече можно решать так.

Пусть $\mathfrak{Z}-$ один из классов $\mathfrak{L}_{\mathrm{M}}^{\mathrm{K}}, \mathfrak{2}_{\mathrm{M}}^{\sigma}$ и $\mathfrak{L}_{\mathrm{M}}$. Чтобы вычислить предикаты $\mathbf{M}_{\mathfrak{L}}^{0}$ и $\mathbf{M}_{\mathfrak{L}}^{1}$ возьмем в качестве коллектива автоматов второго типа автоматы, которые остаются все время поблизости от вершины, в которую они помешены (можем предположить, например, что в любой момент времени все камни этого коллектива лежат в этой вершине и что один из автоматов этого коллектива, не являюшийся камнем, обязательно время от времени посешает указанную вершину). В качестве коллектива первого типа берем тот, который обходит указанный класс лабиринтов. К алгоритму обхода для этого класса добавим еще указание, что в случае встречи с каким-то из автоматов второго коллектива, в том числе и с некоторым его камнем, автомат первого коллектива, который осуществил эту встречу, должен остановиться. Ясно, что в этом случае встреча коллективов произойдет. Правда, логически возможна и ситуация, когда два коллектива, каждый из которых не обходит рассматриваемый класс лабиринтов, тем не менее встречаются в любом лабиринте этого класса. Так получается для класса $\mathfrak{L}_{\mathrm{M}}^{\mathrm{K}}$, доказано даже, что $\mathbf{M}_{\mathfrak{L}_{\mathrm{M}}}^{2}(1,1)=1$. Поскольку для любого автомата, то есть для любого коллектива типа $(1,0)$, существует правильная ловушка в $\mathfrak{Q}_{\mathrm{M}}^{\mathrm{K}}$, легко удостовериться, что $\mathbf{M}_{\mathfrak{S}_{\mathrm{M}}^{\mathrm{k}}}^{0}(1,0,1,0)=0$. Значит, справедливы следующие утверждения.

Теорема 9 ([55]). Для предикатов $\mathbf{M}_{\mathcal{L}_{\mathrm{M}}}^{1} u \mathbf{M}_{\mathcal{L}_{\mathrm{M}}}^{2}$ справедливы соотношения

$$
\mathbf{U}\left[\mathbf{M}_{\mathfrak{L}_{M}^{K}}^{1}\right]=\mathbf{U}\left[\mathbf{M}_{\mathcal{L}_{M}^{K K}}^{2}\right]=\{(1,1),(2,0)\} \text {. }
$$

Теорема 10. Для предиката $\mathbf{M}_{\mathcal{L}_{\mathcal{M}}^{k}}^{0}$ справедливо равенство

$$
\mathbf{U}\left[\mathbf{M}_{\mathcal{Y}_{M}}^{0}\right]=\{(1,2,1,0),(1,0,1,2),(1,1,1,1)(2,0,1,0),(1,0,2,0)\} .
$$

Отметим, что для классов $\mathfrak{L}_{\mathrm{M}}^{6}$ и $\mathfrak{L}_{\mathrm{M}}$ нет полного описания рассмотренных предикатов встречи.

Говорим, что $n$-мерный лабиринт $L$ является циклическим, если он конечен и степень всех вершин графа $\overline{G(L)}$ равна двум. Обозначим через $\mathfrak{L}_{\text {мц }}$ класс всех циклических плоских мозаичных лабиринтов. В [44] установлено, что $\mathbf{M}_{\mathfrak{Z}_{\text {мu }}^{2}}^{2}(1,1)=1$. Там же показано, 
что если в качестве класса $\mathfrak{L}$ берется класс всех деревьев из $\left(\Re_{R}^{+}(d), d \geqslant 3\right.$, то также имеет место утверждение $\mathbf{M}_{\mathfrak{Q}}^{2}(1,1)=1$; отметим, что здесь имеются в виду коллективы $R$-автоматов, состоящие из одного автомата и одного вершинного камня. Считаем, что этот результат требует проверки.

Кроме того, в [44] рассматриваются случаи, когда 2 состоит либо только из одномерной ленты, либо только из лабиринта $\mathbf{Z}^{2}$. В первом случае показано, что

$$
\mathbf{M}_{\mathfrak{L}}^{0}(1,0,1,1)=1, \quad \mathbf{M}_{\mathfrak{L}}^{1}(1,0)=0, \quad \mathbf{M}_{\mathfrak{I}}^{2}(1,1)=1 ;
$$

во втором случае показано, что

$$
\mathbf{M}_{\mathfrak{L}}^{0}(1,0,1,2)=\mathbf{M}_{\mathfrak{l}}^{0}(1,2,1,0)=0, \quad \mathbf{M}_{\mathfrak{L}}^{1}(1,2)=1, \quad \mathbf{M}_{\mathfrak{L}}^{2}(1,2)=0, \quad \mathbf{M}_{\mathfrak{l}}^{2}(1,3)=1 .
$$

Равенство $\mathbf{M}_{\mathfrak{I}}^{1}(1,2)=1$ впервые доказано в [39]; в [44] дан более простой алгоритм для доказательства этого факта, который также обеспечивает встречу за меньшее время, чем алгоритм, приведенный в [39].

В контексте задачи о встрече, когда \& состоит либо только из одномерной ленты, либо только из лабиринта $\mathbf{Z}^{2}$, наряду с коллективами автоматов в [44] рассматривались и некоторые другие системы автоматов и их обобщений; автоматам было разрешено иметь счетчики и даже возможность оставлять метки в вершинах. Предикаты встречи, которые по смыслу отвечают выше введенным, будем обозначать здесь теми же символами. Прежде всего ясно, что две одинаковые копии автоматов с произвольным количеством счетчиков никогда не встретятся ни на одномерной ленте, ни в лабиринте $\mathbf{Z}^{2}$. В случае, когда 2 состоит либо только из одномерной ленты, либо только из лабиринта $\mathbf{Z}^{2}$, справедливы следующие результаты ([44]): для автомата с одним счетчиком значение предиката $\mathbf{M}_{\mathfrak{L}}^{1}$ равно нулю; для коллектива из двух автоматов, у одного из которых два счетчика, значение предиката $\mathbf{M}_{\mathfrak{L}}^{0}$ равно единице.

Если позволить автоматам делать метки в вершинах лабиринтов (при этом, конечно, предполагаем, что автоматы-камни этого не могут делать), то в случае, когда $\mathfrak{Z}=\mathbf{Z}^{2}$, установлено, что $\mathbf{M}_{\mathfrak{\Omega}}^{2}(1,2)=0$ (см. [44]).

В некоторых из вышеприведенных случаев в [44] устанавливаются оценки, верхние или нижние, минимального времени, в течении которого должна обязательно произойти встреча хотя бы одной пары коллективов, для которых значение соответствующего предиката равно единице. Эти оценки выражаются через расстояние между клетками, в которые коллективы помещаются в начальный момент.

Задача о встрече допускает вариации за счет различных допущений относительно узнаваемости автоматами друг друга, своих и чужих камней и т. п. Так, в [39] для задачи о встрече на плоскости в случаях, когда автоматы распознают свои и чужие камни или же не обладают этим свойством, установлено, что $\mathbf{M}_{\mathbf{Z}^{2}}^{1}(1,2)=1$ (см. также предыдущий абзац). Соответствующие коллективы типа $(1,1)$, построенные в [39], которые всегда встречаются на целочисленной плоскости, могут даже начинать работу в разные моменты времени.

Содержательной является задача о взаимодействии в лабиринтах двух автоматов $\mathfrak{A}_{1}$ и $\mathfrak{U}_{2}$, имитирующих поведение типа хищник-жертва. В $[45,46]$ автоматы $\mathfrak{U}_{1}$ и $\mathfrak{P}_{2}$ взаимодействуют в конечных плоских шахматных лабиринтах, имеющих вид квадрата, и задача для хищника $\mathfrak{U}_{1}$ - догнать жертву, а для жертвы $\mathfrak{U}_{2}-$ убежать от хищника. В основной постановке данной задачи считается, что $\mathfrak{U}_{1}$ и $\mathfrak{U}_{2}$ делают ходы поочередно и что $\mathfrak{A}_{1}$ догнал $\mathfrak{U}_{2}$, если $\mathfrak{A}_{1}$ и $\mathfrak{A}_{2}$ оказались в какой-то момент на соседних полях. Установлено, что при любых $l, n \in \mathbf{N}$ сушествует $\mathfrak{U}_{l}$ с числом состояний $O\left(n l^{2}\right)$, который догоняет за 
время $O\left(n l^{4}\right)$ любой $\mathfrak{A}_{2}$ с числом состояний, не большим $n$, в квадрате со стороной, не большей $l$, при любом стартовом положении автоматов $\mathfrak{A}_{1}$ и $\mathfrak{U}_{2}[45,46]$. Там же установлено, что не существует автомата $\mathfrak{A}_{1}$, догоняющего любой $\mathfrak{U}_{2}$ в произвольном квадрате с фиксированной длиной стороны $l, l \geqslant 8$. Первый результат обобщается на случай различных обзоров и скоростей $\mathfrak{A}_{1}$ и $\mathfrak{H}_{2}$. Установлена алгоритмическая разрешимость свойства хищника догонять любую жертву по указанным параметрам.

\section{4. Распознавание свойств лабиринтов с помощью автоматов}

Приведем результаты, связанные с задачей анализа для коллективов автоматов.

Пусть $\mathbf{P}(x)$ - некоторый предикат на множестве лабиринтов $\mathfrak{Q}$, и пусть $\mathscr{A}-$ некоторая лабиринтная машина (автомат, коллектив автоматов, автомат со счетчиком и тому подобное), допустимая для лабиринтов множества $\stackrel{2}{2}$. Некоторое множество состояний $Q_{0}$ машины $\mathscr{A}$ выделено, и его элементы называются заключительными состояниями. Будем говорить, что $\mathscr{A}$ вычисляет $\mathbf{P}$, если при его запуске в любой лабиринт $L \in \mathfrak{Q}$ происходит переход машины $\mathscr{A}$ в некоторое из своих заключительных состояний (в этом случае также говорим, что $A$ принимает $L$ ) только тогда, когда $\mathbf{P}(L)=1$. Если множество заключительных состояний $Q_{0}$ можно представить как дизъюнктное объединение двух множеств $Q_{1}$ и $Q_{2}$ и при этом машина $\mathscr{A}$ переходит в состояние из $Q_{1}$, когда $\mathbf{P}(L)=0$, а в состояние из $Q_{2}$, когда $\mathbf{P}(L)=1$, то говорим, что $\mathscr{A}$ сильно вычисляет $\mathbf{P}$. Задачу, состоящую в выяснении, вычисляет (сильно вычисляет) или нет машина $\mathscr{A}$ предикат $\mathbf{P}$ называем задачей анализа.

Сделаем несколько замечаний. Выше предполагалось, что машина $\mathscr{A}$ является детерминированной. Если она недетерминирована, то для того, чтобы принять некоторый лабиринт, она должна перейти в некоторое из заключительных состояний хотя бы при одном из возможных вычислений. Для случая, когда в качестве лабиринтной машины выступает коллектив автоматов (без печати), введем следующее определение. Будем говорить, что некоторый предикат является (сильно) $(i, j)$-вычислимым, если существует коллектив типа $(i, j)$, который (сильно) вычисляет этот предикат. Также ясно, что в случае вычислимости предиката $\mathbf{P}$ коллективом из $k$ автоматов без печати, факт принятия некоторого $n$-вершинного лабиринта происходит обязательно за время, не большее $(\mathrm{nm})^{k}$, где $m$ - максимум по числу состояний всех автоматов рассматриваемого коллектива.

Возможным путем описания предикатов, вычислимых с помощью коллективов автоматов, является построение соответствующей алгебры над вычислимыми предикатами, сохраняющей свойство вычислимости. Пример такой алгебры для множества всех регулярных событий принадлежит С. Клини [55]. Она содержит три операции: объединение, конкатенацию и итерацию множеств слов. В [20] устанавливается, что операции конкатенации и итерации для лабиринтов, вообще говоря, нарушают вычислимость. Точнее, пусть $\Omega$ - некоторое конечное множество символов. Обозначим через $\Omega^{(2)}$ множество всех конечных плоских шахматных лабиринтов прямоугольного вида, вершины которых отмечены символами из $\Omega$. В [20] приводятся примеры подмножеств из $\{0,1\}^{(2)}$, для которых соответствующие предикаты вычислимы некоторыми автоматами, а конкатенация (склеивание по строкам лабиринтов из $\{0,1\}^{(2)}$, имеющих одну и ту же высоту) и итерация этих подмножеств лабиринтов не всегда образуют подмножество из $\{0,1\}^{(2)}$, для которого соответствующий предикат является вычислимым каким-то автоматом.

На автоматы, допустимые для класса $\Omega^{(2)}$, можно смотреть как на естественное обоб- 
щение одномерных двусторонних автоматов на двумерный случай. Тогда естественным обобщением на двумерный случай одномерных односторонних автоматов будут допустимые для $\Omega^{(2)}$ так называемые трехсторонние автоматы, то есть автоматы, которые не могут двигаться вверх, а только налево, направо и вниз. Известно, что в одномерном случае односторонние автоматы не являются менее сильными (в смысле вычислимости) по сравнению с двухсторонними. Интересно, что в двумерном случае трехсторонние автоматы оказываются менее сильными, чем (четырехсторонние) автоматы (подробнее см. в $[20,22])$. Обозначим классы детерминированных и недетерминированных трехсторонних автоматов, соответственно, через $C$ [2TR-DA] и $C$ [2TR-NA]. В случае, когда $\Omega$-- однобуквенный алфавит, вместо $C$ [2TR-DA] ( $C$ [2TR-NA]) пишем $C$ [2TR-DA(0)] ( $C$ [2TR-NA(0)]). Инициальный трехсторонний (недетерминированный) детерминированный автомат принимает некоторый лабиринт $L$ из $\Omega^{(2)}$, если, начиная с левого верхнего угла лабиринта $L$, доходит до некоторого поля самой нижней строки, оказываясь при этом в некотором из своих заключительных состояний. Для любого автомата $\mathfrak{A}$ из одного из введенных классов через $\mathbf{P}(\mathfrak{U})$ обозначим предикат, который вычисляет $\mathfrak{U}$ на множестве $\Omega^{(2)}$, а через $\mathbf{R}(\mathfrak{U})$ - область истинностности этого предиката. Для любых трехсторонних автоматов $\mathfrak{U}_{1}, \mathfrak{U}_{2}$, и $\mathfrak{U}_{3}$ одного из введенных классов нас интересует вопрос, выполнены ли соотношения $\mathbf{R}\left(\mathfrak{H}_{1}\right) \subseteq \mathbf{R}\left(\mathfrak{H}_{2}\right), \mathbf{R}\left(\mathfrak{H}_{1}\right) \cap \mathbf{R}\left(\mathfrak{H}_{2}\right)=\varnothing$ и $\mathbf{R}\left(\mathfrak{H}_{1}\right)=\mathbf{R}\left(\mathfrak{H}_{2}\right)$, а также $\mathbf{R}\left(\mathfrak{A}_{3}\right)=\Omega^{(2)}$ и $\mathbf{R}\left(\mathfrak{A}_{3}\right)=\varnothing$; соответствуюшие проблемы называем проблемами $\mathbf{R}_{1} \subseteq \mathbf{R}_{2}, \mathbf{R}_{1} \cap \mathbf{R}_{2}=\varnothing$, $\mathbf{R}_{1}=\mathbf{R}_{2}, \mathbf{R}=\Omega^{(2)}$ и $\mathbf{R}=\varnothing$.

В [24] показывается, что для класса $C\left[2 \mathrm{TR}-\mathrm{DA}(0)\right.$ ] задачи $\mathbf{R}_{1} \cap \mathbf{R}_{2}=\varnothing$ и $\mathbf{R}_{1} \subseteq \mathbf{R}_{2}$ алгоритмически разрешимы. По существу, здесь основным фактом является разрешимость проблемы $\mathbf{R}_{1} \cap \mathbf{R}_{2}=\varnothing$. Разрешимость проблемы $\mathbf{R}_{1} \subseteq \mathbf{R}_{2}$ следует из упомянутого факта и из доказанного в [22] факта, что для любого $\mathfrak{A} \in C[2 \mathrm{TR}-\mathrm{DA}(0)]$ эффективным способом можно построить автомат $\overline{\mathfrak{U}} \in C[2 \mathrm{TR}-\mathrm{DA}(0)]$ такой, что $\mathbf{R}(\overline{\mathfrak{A}})=\Omega^{(2)} \backslash \mathbf{R}(\mathfrak{H})$ (условно назовем это свойство класса $C[2 \mathrm{TR}-\mathrm{DA}(0)]$ свойством его замкнутости по отношению к операции взятия дополнения). Разрешимость проблемы $\mathbf{R}_{1}=\mathbf{R}_{2}$ для класса $C[2 \mathrm{TR}-\mathrm{DA}(0)]$ очевидным образом следует из разрешимости проблемы $\mathbf{R}_{1} \subseteq \mathbf{R}_{2}$ для того же класса. В [24] также показано, что для класса $C$ [2TR-DA] задачи $\mathbf{R}_{1} \cap \mathbf{R}_{2}=\varnothing$ и $\mathbf{R}_{1} \subseteq \mathbf{R}_{2}$ неразрешимы (на самом деле неразрешимость первой задачи прямо влечет неразрешимость второй). Там же доказывается, что существуют такие предикаты на множестве $\Omega^{(2)}$, которые вычисляются недетерминированными трехсторонними автоматами и не вычисляются детерминированными трехсторонними автоматами, то есть показано, что недетерминированный вариант этих автоматов сильнее детерминированного. Ранее (в [22]) показано, что для классов $C[2 \mathrm{TR}-\mathrm{DA}(0)]$ и $C[2 \mathrm{TR}-\mathrm{NA}(0)]$ задача $\mathbf{R}=\varnothing$ алгоритмически разрешима. Там же показано, что задача $\mathbf{R}=\Omega^{(2)}$ также алгоритмически разрешима для класса $C[2 \mathrm{TR}-\mathrm{DA}(0)]$ (это утверждение является на самом деле прямым следствием предыдущего и замкнутости этого класса по отношению к операции взятия дополнения), а для класса $C$ [2TR-NA] задачи $\mathbf{R}=\Omega^{(2)}, \mathbf{R}_{1} \subseteq \mathbf{R}_{2}$ и $\mathbf{R}_{1}=\mathbf{R}_{2}$ алгоритмически не разрешимы.

Для любого $L \in \Omega^{(2)}$ обозначим через $l_{1}(L)$ число его строк, а через $l_{2}(L)$ число его столбцов. Пусть $f: \mathbf{N} \rightarrow \mathbf{N}$ - произвольная (натуральная) функция. Пусть $\mathfrak{A}$ - некоторый недетерминированный автомат, допустимый для класса $\{0\}^{(2)}$. Предположим, что смысл обозначений $\mathbf{P}(\mathfrak{U})$ и $\mathbf{R}(\mathfrak{U})$ остается прежним. Говорим, что автомат $\mathfrak{A}$ вычисляет функцию $f$, если

$$
\mathbf{R}(\mathfrak{U})=\left\{L \in\{0\}^{(2)} \mid l_{1}(L)=f(n) \text { и } l_{2}(L)=n \text { для некоторого } n \in \mathbf{N}\right\} .
$$

Функщия $f$ является невычислимой (недетерминированным) автоматом, если нет такого 
автомата, который ее вычисляет.

В [21] показывается, что функции $f_{1}(n)=n^{2}, f_{2}(n)=k^{n}$ и $f_{3}(n)=n$ ! невычислимы автоматом. Другими словами, предикаты $\mathbf{P}_{1}, \mathbf{P}_{2}$ и $\mathbf{P}_{3}$ на множестве $\{0\}^{(2)}$, области истинностности которых суть множества всех лабиринтов из $\{0\}^{(2)}$ размеров $n^{2} \times n, k^{n} \times n$ и $n ! \times n$, соответственно, не являются вычислимыми недетерминированными автоматами. До сих пор неизвестно какое-либо достаточно полезное необходимое условие, которому должны удовлетворять вычислимые натуральные функции.

В [31] изучается вопрос о том, какие классы размеченных лабиринтов с выделенной границей распознаваемы недетерминированными автоматами в том смысле, что автомат находит в них выход, переходя в заключительное состояние. Введено специальное кодирование таких лабиринтов, для которого показано, что в возникающем формальном языке описания лабиринтов распознаваемы только те лабиринты, которые кодируются регулярными языками. Остановимся более подробно на этом результате.

Берется конечное множество различных символов $s_{1}, s_{2}, \ldots, s_{m}$. Для каждого $s_{i}, i=1, \ldots, m$, вводится новый символ $s_{i}^{-1}$ (обратный элемент для $s_{i}$ ). Множество $S=\left\{s_{1}, \ldots, s_{m}, s_{1}^{-1}, \ldots, s_{m}^{-1}\right\}$ называется множеством образующих. Имеется и некоторое конечное множество $R$ слов в алфавите $S$; эти слова можно назвать (согласно языку теории свободных групп) отношениями. Множеству $R$ принадлежат пустое слово $\Lambda$ и все слова вида $s_{i} s_{i}^{-1}, s_{i}^{-1} s_{i}$ и $s_{i} s_{j} s_{i}^{-1} s_{j}^{-1}$. На множестве $S^{*}$ определяется отношение эквивалентности $\sim$ следующим способом: слова $w_{1}, w_{2} \in S^{*}$ называются $R$-эквивалентными, $w_{1} \sim w_{2}$, если существует последовательность слов $u_{1}, u_{2}, \ldots, u_{n} \in S^{*}$ такая, что $w_{1}=u_{1}, w_{2}=u_{n}$ и для любого $1 \leqslant i \leqslant n-1$ слово $u_{i+1}$ получается из слова $u_{i}$ таким способом, что или вставляется в $u_{i}$ некоторое слово из $R$, или в нем зачеркивается некоторое подслово (заменяется пустым словом), которое равно некоторому слову из $R$. На фактор-множестве $S^{*} / \sim$ введем операцию - следующим способом: $[u] \cdot[v]=[u v]($ здесь $[x]-$ класс эквивалентности элемента $x$ в $S^{*}$ по отношению к $\left.\sim\right)$. Легко удостовериться, что пара $\left(S^{*} / \sim, \cdot\right)$ является абелевой группой (абелевой конечно представимой группой - АКП-группой); обозначим ее через $(S ; R)$. Группа такого рода может описывать структуру вида регулярной (однородной) решетки (примером такой решетки является целочисленное $n$-мерное пространство) следующим образом. Вершинам этой решетки (она понимается как граф) соответствуют элементы этой группы, а всевозможным основным направлениям (по которым переходим из некоторой точки в ее соседние вершины) - элементы из $S$. Тогда основным циклам отвечают слова из $R$. Например, пространство $\mathbf{Z}^{2}$ описывается группой $G_{2}=\left(s_{1}, s_{2} ; s_{1} s_{1}^{-1}, s_{1}^{-1} s_{1}, s_{2} s_{2}^{-1}, s_{2}^{-1} s_{2}, s_{1} s_{2} s_{1}^{-1} s_{2}^{-1}\right)$, при этом $s_{1}$ и $s_{2}$ интерпретируем как направления е и $\mathbf{n}$, соответственно, а элементы этой группы как целочисленные точки из $\mathbf{Z}^{2}$. Группа $G_{2}$ полностью алгебраически описывает топологическую природу двумерной целочисленной решетки. Таким способом, по утверждению автора статьи [31], можно получить все пространства, которые представляют интерес для теории распознавания образов.

Пусть дана некоторая АКП-группа $G$ (тем самим, дана соответствующая ей решетка), и пусть $V$ - некоторое конечное множество. Пару вида $(G, V)$ назовем дискретным пространством. Под образом дискретного пространства $(G, V)$ понимается любой конечный связный подграф дискретного пространства, у которого вершины отмечены элементами множества $V$; обозначим через $\Pi(G, V)$ множество всех образов дискретного пространства $(G, V)$. Считаем, что этот образ вложен в полное дискретное пространство, причем любая клетка, не принадлежащая образу, маркирована буквой $\beta \notin V$, если она не является соседней ни одной клетке образа и маркирована буквой $B \notin V$, если она является соседней хотя бы одной клетке образа (лежит на границе образа). Следователь- 
но, образ - это размеченный лабиринт с отмеченной границей. Также фиксированы две точки образа, взятого вместе с границей, которые объявляются начальной и конечной. Далее, дан некоторый конечный алфавит $\Sigma$, которым кодируются элементы множества $\mathfrak{B}=(V \cup\{B\}) \times(S \cup\{\Lambda\})$ (множества $\Sigma$ и $\mathfrak{R}$ равномощны). Конечные последовательности элементов множества $\mathfrak{B}$ назовем помеченными путями. Говорим, что помеченный путь $\left(b_{1}, \omega_{1}\right),\left(b_{2}, \omega_{2}\right), \ldots,\left(b_{m}, \omega_{m}\right)$ с началом в точке $v$ данного дискретного пространства посещает точки $v, v \omega_{1}, v \omega_{1} \omega_{2}, \ldots, v \omega_{1} \omega_{2} \ldots \omega_{m}$ и приписывает им, соответственно, метки $b_{1}, b_{2}, \ldots, b_{m}$, если метка вершины $v$-символ $b_{1}$, а метка вершины $v \omega_{1} \omega_{2} \ldots \omega_{k}$, $1 \leqslant k \leqslant m-1$, пути $v, v \omega_{1}, v \omega_{1} \omega_{2}, \ldots, v \omega_{1} \omega_{2} \ldots \omega_{m}-$ символ $b_{k+1}$. Каждый язык $L \subseteq \Sigma^{*}$ над алфавитом $\Sigma$ задает естественным путем некоторое множество помеченных путей $\mathfrak{B}(L)$. Пусть $\pi$ - некоторый образ, $u_{0}$ - его начальная вершина, а $u_{1}-$ его конечная вершина. Тогда ясно, что любой путь в $\pi$, который ведет из точки $u_{0}$ в точку $u_{1}$, определяет вполне естественным образом некоторый помеченный путь; обозначим через $C_{\pi}$ все таким способом полученные помеченные пути (ясно, что $C_{\pi}-$ непустое множество для любого $\pi)$. Под множеством образов $P(L,(G, V))$, которое определяет язык $L \subseteq \Sigma^{*}$ в $(G, V)$, подразумеваем множество

$$
P(L,(G, V))=\left\{\pi \mid \pi \in \Pi(G, V) \wedge \mathfrak{B}(L) \cap C_{\pi} \neq \varnothing\right\} .
$$

Поскольку любой образ - это размеченный по вершинам лабиринт, в нем можно изучать поведение соответствующих недетерминированных допустимых автоматов без печати, так называемых $m$-сторонних конечных автоматов (число $m$ указывает на число элементов множества $S$ ), у которых поле зрения охватывает только текущую вершину и которые могут двигаться по всем основным направлениям. Пусть $\mathfrak{U}$ - такой автомат. Тогда под множеством образов, которое определяет этот автомат, понимаем все образы, в которых автомат $\mathfrak{A}$, помешенный в начальную точку этого образа, находит выход (хотя бы одна его траектория оканчивается в конечной точке этого образа). В [31] показано, что класс множеств образов, которые определяют регулярные языки над $\Sigma$, равен классу множеств образов, которые определяют $m$-сторонние конечные автоматы.

В [47] рассмотрено поведение автоматов в лабиринтах с точки зрения характеризации их траекторий в лабиринтах как формальных языков. Для автоматов вводятся два типа эквивалентности: согласно первому, автоматы считаются эквивалентными, если в лабиринтах они порождают одно и то же множество траекторий, согласно второму если эти множества траекторий отличаются мало. Изучаемая проблема состояла в следуюшем: по любой заданной паре автоматов решить вопрос их эквивалентности (в обоих смыслах). Было выделено несколько семейств лабиринтов (таких, как прямоугольники в случае плоскости, лабиринты, полученные склеиванием по строкам прямоугольников, не обязательно одинаковых по высоте, и др.) как модельных для изучения этой задачи. Для этих семейств данная проблема решена. Установлена ее алгоритмическая разрешимость.

В $[33,34]$ рассматривается машина Тьюринга, как детерминированная, так и недетерминированная, у которой несколько рабочих лент и одна входная лента (многоленточный вариант машины Тьюринга). Входная лента содержит всегда входное слово в конечном входном алфавите $\Sigma$, и входное слово отделено от остальной части входной ленты двумя символами (левым и правым ограничителем), не принадлежащими $\Sigma$. В любой клетке рабочих лент может находиться некоторый символ конечного рабочего алфавита $Г$ (пустой символ $\Lambda$ принадлежит алфавиту $Г$ ). Как обычно, делаются следующие предположения. У любой ленты по одной головке, у входной - входная головка, у рабочих лент - рабочие головки. Входная головка только читает, а рабочие могут и читать и писать; при этом любая головка (как входная, так и рабочая) может либо двигаться независимо от других 
головок на одну клетку влево или вправо, либо оставаться на месте. У машины конечное множество состояний, и она так устроена, что во время ее работы входная головка никогда не покидает сегмент входной ленты, содержащий входное слово и ограничители. В любой момент дискретного времени машина читает обозреваемые головками клетки всех лент и решает в зависимости от полученной информации и своего состояния, что написать в них (это относится только к клеткам рабочих лент) и куда головкам двигаться, и при этом переходит в новое состояние. У машины есть одно начальное состояние и некоторое количество заключительных состояний. Говорим, что машина допускает некоторое входное слово (оно предоставляется машине через входную ленту), если для этой машины, начинающей работу с начального состояния, с пустыми рабочими лентами (во все клетки рабочих лент вписан пустой символ $\Lambda$ ) и с входной головкой, сканирующей левый ограничитель входного слова, существует хотя бы одно вычисление (в детерминированном случае может сушествовать только одно такое вычисление), которое переводит машину в некоторое из заключительных состояний. Описывая результаты работ $[33,34]$, мы будем иметь в виду именно такой вариант машины Тьюринга, то есть, или детерминированную многоленточную машину Тьюринга (ДМТ), или недетерминированную многоленточную машину Тьюринга (НMT).

Пусть $f(n)$ - некоторая натуральная функция натуральной переменной и $\mathbf{A} \subseteq \Sigma^{*}-$ некоторый язык в алфавите $\Sigma$. Говорим, что А определяется (или распознается) некоторой (НМТ) ДМТ с длиной рабочей зоны $f(n)$, если существует (НMT) ДМТ $\mathfrak{M}$ такая, что $\mathfrak{M}$ допускает любое слово $\alpha \in \mathbf{A}$ и не допускает ни одного слова из $\Sigma^{*} \backslash \mathbf{A} ;$ у $\mathfrak{M}$ существует хотя бы одно вычисление для любого слова $\alpha \in \mathbf{A}$, при котором любая из головок машины обозревает не более $f(|\alpha|)$ клеток.

В [33] доказано, что если некоторое множество слов $\mathbf{A} \subseteq \Sigma^{*}$ определяется некоторой НМТ с длиной рабочей зоны $f(n) \geqslant \log _{2} n$, то А определяется некоторой ДМТ с длиной рабочей зоны $[f(n)]^{2}$ (здесь предполагается, что $f(n) \geqslant \log _{2} n$, поскольку в соответствуюшем доказательстве приведенного выше факта требуется, чтобы функция $f(n)$ была конструируемой по емкости). Дальше ставился вопрос, можно ли у указанной ДМТ уменьшить длину рабочей зоны, например, до минимальной $f(n)$. Другими словами, имеет ли место следуюшее утверждение: для любого конечного алфавита $\Sigma$, любого $\mathbf{A} \subseteq \Sigma^{*}$ и любой функции $f(n) \geqslant \log _{2} n$, если А определяется некоторой НМТ с длиной рабочей зоны $f(n)$, то А определяется некоторой ДМТ с длиной рабочей зоны $f(n)$ ? Назовем соответствующую проблему проблемой $f(n)$-ДМТ $=f(n)$-НМТ?.

НМТ с длиной рабочей зоны $f(n)=n$ называется линейно ограниченным автоматом (LBA), а ДМТ с такой же длиной рабочей зоны называется детерминированным линейно ограниченным автоматом (DLBA). Как известно, класс контекстных (или контекстнозависимых) языков точно определяется классом всех LBA. Открытым остается вопрос, распознается ли любой контекстный язык некоторым DLBA, то есть проблема совпадения классов языков, определяемых LBA и DLBA, называемая проблемой DLBA=LBA?. Ясно, что положительный ответ на проблему $f(n)$-ДМТ $=f(n)$-НМТ? влечет за собою положительный ответ на проблему DLBA=LBA?.

В связи с проблемой $f(n)$-ДМТ $=f(n)$-НМТ? исследовалась следующая лабиринтная задача. В [34] рассматривались лабиринты вида орграфов, удовлетворяющих следующим условиям:

(a) существует контур, содержащий все вершины орграфа, каждая дуга которого отмечена символом 0 ;

(b) из каждой вершины орграфа исходят еще точно по две дуги, отмеченные соответст- 
венно символами 1 и 2;

(c) в орграфе отмечена одна вершина в качестве начальной и группа вершин в качестве конечных, причем предполагается, что допустимые автоматы (коллективы автоматов) по этим меткам могут узнавать, что текущая вершина (любая из текущих вершин) является начальной или конечной вершиной.

Такой лабиринт называется ниточным, если в нем существует путь, который идет по дугам лабиринта, отмеченным символами 1 или 2 , и который ведет из начальной в какую-то из конечных вершин. Показано, что проблема $f(n)$-ДМТ $=f(n)$-НМТ? эквивалентна следующей лабиринтной проблеме: сушествует ли, хотя бы для одного $j \in \mathbf{N}$, коллектив автоматов типа $(1, j)$, вычисляющий предикат $\mathbf{P}_{\mathrm{th}}$, у которого областью истинности является класс всех ниточных лабиринтов? Отметим, что из предыдущего следует, что сушествование коллектива типа $(1, j)$, который вычисляет предикат $\mathbf{P}_{\text {th }}$, приводит к положительному ответу на проблему LBA=NLBA?.

В [5] установлено, что не существует коллектива типа $(1,2)$, вычисляюшего предикат $\mathbf{P}_{\text {th. }}$.

В [29] рассматриваются два типа автоматов с печатью (машин Тьюринга), допустимых для размеченных конечных плоских шахматных лабиринтов. Эти лабиринты считаются вложенными в $\mathbf{Z}^{2}$ таким способом, что точки, которые не принадлежат лабиринтам, отмечены одним специальным символом \# (назовем эти точки \#-клетками), которым не отмечаются точки лабиринтов; такие лабиринты в [29] названы массивами (см. ниже). Автомату первого типа дано название массивом ограниченного автомата (MOA), причем рассматривается его детерминированный вариант (ДМОА), а второй тип коротко обозначается через \#-ДМОА. Первый тип - это просто автомат с печатью, он движется по лабиринту, может менять метки его клеток, но не ходит по \#-клеткам, поскольку они не принадлежат данному лабиринту. Автомат второго типа может делать все, что делает автомат первого типа, но ему дополнительно разрешено выходить за пределы лабиринта (ходить по \#-клеткам), хотя там ему не позволено печатать. Показано, что классы вычислимых предикатов $\mathbf{P}$ для этих типов автоматов совпадают. Заметим, что для непечатающих вариантов машин ДМОА и \#-ДМОА это утверждение неверно: второй тип автоматов сильнее первого.

Ряд работ посвящен изучению лабиринтов с помощью представления их словами формальных языков. Некоторые алгебраические характеристики общего вида взаимодействия автоматов и лабиринтов приводятся в [28]. В этой статье вводится понятие акцегтора массивов Тьюринга (АМТ), причем под (двумерным) массивом понимается размеченная целочисленная плоскость. При этом предполагается, что соответствующая разметка всегда имеет следующий вид: среди (временных) меток имеется один специальный символ \# такой, что множество всех полей, не помеченных символом \#, определяет в $\mathbf{Z}^{2}$ подлабиринт, который представляет собою (размеченный) конечный шахматный лабиринт; обозначим этот лабиринт для данного массива $M$ через $L_{M}$. Тогда, согласно нашей терминологии, AMT является просто недетерминированным конечным автоматом с печатью, который двигается по такой плоскости. Никаких дополнительных ограничений на АМТ относительно печати не накладывается. Он может писать даже в клетках, в которых был символ \# (предварительно его стирая). Единственное ограничение касается стартовой точки: предполагается, что она всегда является клеткой лабиринта $L_{M}$. Как и выше, предполагается, что у АМТ есть одно начальное и некоторое множество заключительных состояний. Кроме того, вводятся грамматики массивов (ГМ) (двумерные аналоги формальных грамматик), которые порождают классы (языки) массивов (лабиринтов); эти классы назовем 
формальными языками лабиринтов. Обозначим для АМТ $\mathfrak{A}$ через $\mathbf{R}(\mathfrak{A})$ класс массивов, которые принимает $\mathfrak{A}$, а для ГM (S) через Lang((S) язык, который она порождает. B [28] показано, что для любого АMT $\mathfrak{A}$ существует ГM (S) такая, что $\operatorname{Lang}((\mathbb{S})=\mathbf{R}(\mathfrak{U})$, и обратно для любой ГМ (S) существует АMT $\mathfrak{A}$ такой, что $\mathbf{R}(\mathfrak{U})=\operatorname{Lang}(\mathbb{S})$. Вводится подкласс класса всех ГМ такой, что аналогичное утверждение имеет место для гюбой грамматики из него и любого недетерминированного МОА.

В [2] рассматривается класс всех квадратных лабиринтов из $\{0,1\}^{(2)}$. Изучается вопрос о том, какие предикаты, определенные на этом классе, вычислимы с помощью коллективов автоматов. Показано, что для всякого $k$ существует коллектив типа $(1,2 k+4)$, вычисляющий те предикаты, которые не вычисляются ни одним коллективом типа $(1, k)$; этот факт оказывается верным и при сравнении типов $(1,2)$ с $(1,1)$ и $(1,1)$ с $(1,0)$.

Обозначим через $\mathbf{P}_{4}, \mathbf{P}_{5}(n), n \in \mathbf{N}_{0}$, и $\mathbf{P}_{6}$ предикаты на множестве $\stackrel{2}{w}_{w}^{\kappa}$ всех конечных плоских шахматных лабиринтов, области истинности которых суть, соответственно, все циклические лабиринты из $\mathfrak{2}_{w}^{2}$, которые имеют точно $n$ целочисленных дыр и у которых хотя бы одна нецелочисленная дыра. В [30] установлено, что предикаты $\mathbf{P}_{4}$ и $\mathbf{P}_{5}(n)$ при любом $n \in \mathbf{N}_{0}$ не являются вычислимыми одним автоматом. С другой стороны, в [35] показано, что предикат $\mathbf{P}_{4}$ является сильно $(1,1)$-вычислимым, предикат $\mathbf{P}_{5}(0)$ - сильно $(1,2)$-вычислимым, а в [8] установлено, что предикат $\mathbf{P}_{5}(n)$ является сильно $(1,2)$-вычислимым для любого $n \in \mathbf{N}_{0}$. Также в [35] показано, что предикат $\mathbf{P}_{6}$ является $(1,1)$-вычислимым и сильно $(1,2)$-вычислимым (очевидно, что сильная $(1,2)$-вычислимость предиката $\mathbf{P}_{5}(0)$ влечет сильную $(1,2)$-вычислимость предиката $\mathbf{P}_{6}$, и обратно, сильная $(1,2)$-вычислимость предиката $\mathbf{P}_{6}$ влечет сильную $(1,2)$-вычислимость предиката $\left.\mathbf{P}_{5}(0)\right)$.

Пусть $\mathbf{P}_{7}-$ предикат на множестве $\underset{\mathfrak{L}_{\mathrm{w}}^{\mathrm{k}}}{\mathrm{k}}$, у которого областью истинности является класс всех лабиринтов из $\mathfrak{L}_{ш}^{\kappa}$, содержащих простое число вершин, и $\mathbf{P}_{8}-$ предикат на множестве $\mathfrak{L}_{w}^{\kappa}$, У которого областью истинности является класс всех лабиринтов из $\mathfrak{2}_{ш}^{\mathrm{K}}$, содержащих две одинаковые дыры. В [8] установлено, что предикат $\mathbf{P}_{7}$ является сильно $(1,5)$-вычислимым, а предикат $\mathbf{P}_{8}-$ сильно $(1,4)$-вычислимым. Показано, что коллективы типа $(1,2 k+4)$ сильнее коллективов типа $(1, k)$ в том смысле, что конструктивно указываются предикаты, сильно вычислимые коллективами типа $(1,2 k+4)$, но не коллективами типа $(1, k)$. Показано также, что если предикат $\mathbf{P}$ сильно вычислим коллективом типа $(1, k)$, то он сильно вычислим машиной Тьюринга с длиной рабочей зоны $\log _{2} n$, и что если такая двухбуквенная машина с длиной рабочей зоны $z \log _{2} n$ сильно вычисляет некоторый предикат Р, то этот предикат сильно вычисляется некоторым коллективом типа $(1,3 z+3)$, где $n$ число вершин лабиринта (под машиной Тьюринга здесь подразумевается ее обобщенный вариант с одной рабочей лентой, а в качестве входной ленты выступает лабиринт, по которому ходит входная головка; такая машина Тьюринга называется $k$-буквенной, если ее рабочий алфавит состоит из $k$ букв). Также установлено, что если некоторый коллектив типа $(1, k)$ сильно вычисляет некоторый предикат $\mathbf{P}$ и $l \geqslant k$, то не существует алгоритма, который бы для любого коллектива типа $(1, l)$ устанавливал, сильно вычисляет этот коллектив предикат Р или нет.

Применение автоматов в лабиринтах для решения задач распознавания образов, а именно, распознавания текста, является предметом исследований проведенных в работах [68-70]. Предлагаемые автоматам шахматные лабиринты имеют форму цифр (в данных работах автор ограничивается цифрами, но в принципе, можно рассматривать вместо цифр любые буквы некоторого алфавита или подобные двумерные объекты). На самом деле, лабиринты, которые здесь рассматриваются, являются дискретизированными образами цифр. Эти образы формализуются - они представляются в виде дизъюнктных 
объединений некоторых основных блоков, данных в виде односвязных шахматных лабиринтов (то есть плоских шахматных лабиринтов без конечных целочисленных дыр). Набор основных блоков по своим формам конечен, но размеры блоков и изрезанность боковых сторон этих блоков произвольны (здесь боковые стороны даны в виде лестниц с количеством ступенек, которое можно варьировать). Этим формализуется неизбежный шум, который всегда сопровождает процесс распознавания (например, классы основных блоков инвариантны по отношению к сжатиям и растягиваниям в направлениях координатных осей). Для каждой цифры вводится соответствующий предикат на множестве плоских шахматных лабиринтов, областью истинности которого является именно класс лабиринтов, соответствующих этой цифре; обозначим $C_{i}$ предикат, который соответствует цифре $i, 0 \leqslant i \leqslant 9$. Изучается сильная вычислимость этих предикатов, то есть распознаваемость соответствующих цифр автоматами. В [70-72] показано, что предикаты $C_{1}, C_{2}$, $C_{3}, C_{5}$ и $C_{7}$ сильно вычисляются одним автоматом, а предикаты $C_{0}, C_{4}, C_{6}, C_{8}$ и $C_{9}$ являются сильно $(1,1)$-вычислимыми (см. также [30]), но не вычисляются одним автоматом. Для любого из выше данных предикатов в упомянутых работах построены соответствующие автоматы или коллективы автоматов типа $(1,1)$, сильно вычисляющие их. Также оценивается сложность распознавания каждой цифры, даются верхние оценки времени, необходимого для вычисления каждого из предикатов (то есть, для принятия произвольного лабиринта из $n$-сечения области истинности соответствуюшего предиката). Дадим точное определение понятия времени вычисления предиката.

Под $n$-сечением $(n \in \mathbf{N})$ класса лабиринтов $\mathfrak{R}$ понимаем множество

$$
\left.\mathfrak{R}\right|_{n}=\{L \in \mathbb{R}|| V(L) \mid=n\} .
$$

Пусть $\mathfrak{Q}$ - некоторый класс лабиринтов, P - некоторый предикат на $\mathfrak{Q}$ и $\mathcal{M}$ - некоторый класс лабиринтных $\mathfrak{2}$-монстров. Через $M(\mathbf{P})$ - обозначим класс всех монстров из $M$, вычисляющих предикат $\mathbf{P}$, а через $\mathfrak{Q}(\mathbf{P})$ - область истинности предиката $\mathbf{P}$. Далее, пусть $\mathfrak{M} \in \mathcal{M}(\mathbf{P})$ и $L \in \mathfrak{L}(\mathbf{P})$. Через $\mathrm{T}_{\mathbf{P}}(\mathfrak{M}, L)$ обозначим время, за которое монстр $\mathfrak{M}$ принимает лабиринт $L$.

Под временем вычисления предиката Р относительно класса $\mathcal{M}$ понимаем функцию

$$
\mathrm{T}_{\mathbf{P}}(n)=\min \left\{\max \left\{\mathrm{T}_{\mathbf{P}}(\mathfrak{M}, L)|L \in \mathfrak{R}(\mathbf{P})|_{n}\right\} \mid \mathfrak{M} \in \mathcal{M}(\mathbf{P})\right\} ;
$$

причем здесь считаем, что $\max \varnothing=0, \max X=\infty$ для любого неограниченного сверху множества $X \subseteq \mathbf{R}$ и $\min \{\infty\}=\infty$. Часто указание на класс $\mu$ опускаем, поскольку из контекста бывает ясно, о каком именно классе $M$ идет речь.

Вопрос оценки времени вычисления некоторого предиката P примыкает к соответствующей задаче анализа для этого предиката. Заметим, что по отношению к предикатам, которые уже рассматривались в настоящем разделе, этот вопрос является мало исследованным. Также заметим, что проблема оценки времени вычисления данного предиката на множестве лабиринтов $\mathfrak{L}$ тесно примыкает к проблеме оценки $T$-сложности обхода класса 2 [54]: интуитивно ясно, что в большинстве случаев, при достаточно разумных предположениях, монстр должен сначала обойти лабиринт, а затем решить вопрос о его принятии. Сам вопрос оценки $T$-сложности обхода в случае некоторых классов лабиринтов уже затрагивался в ранее упомянутых работах $[13,50,53]$, а в работе [63] он является главным, но применительно к автомату с печатью. Приведем здесь эти результаты.

В [54] рассматривалась $T$-сложность обхода некоторого класса $\mathfrak{Q}$ по отношению к автоматам, допустимым для $\mathcal{Q}$. Обобщим это понятие на случай произвольных монстров.

Пусть $\mathfrak{L}-$ некоторый класс лабиринтов и $\mathcal{M}$ - некоторый класс лабиринтных $\mathfrak{L}$-монстров. Обозначим через $\operatorname{Un}_{\mathcal{M}}(\mathfrak{Q})$ множество всех универсальных обходчиков из $\mathcal{M}$ 
для $\mathfrak{L}$, то есть множество всех монстров из $\mathcal{M}$, обходящих любой лабиринт из $\mathfrak{L}$. Далее, пусть $\mathfrak{M} \in \operatorname{Un}_{\mathcal{M}}(\mathfrak{L})$ и $L \in \mathfrak{L}$. Через $\mathrm{T}(L, \mathfrak{M})$ обозначим время, за которое монстр $\mathfrak{M}$ обходит лабиринт $L$. Пусть

$$
\mathrm{T}(\mathfrak{L}, n ; \mathfrak{M})=\max \left\{\mathrm{T}(L, \mathfrak{M})|L \in \mathfrak{Q}|_{n}\right\}
$$

для любого $\mathfrak{M} \in \mathrm{Un}_{\mathcal{M}}(\mathfrak{L})$ и $n \in \mathbf{N}$. Также положим

$$
\begin{aligned}
\mathrm{T}(\mathfrak{Q}, n ; m) & =\min \left\{\mathrm{T}(\mathfrak{Q}, n ; \mathfrak{M})\left|\mathfrak{M} \in \mathrm{Un}_{\mathcal{M}}(\mathfrak{Q}) \wedge\right| Q_{\mathfrak{M}} \mid=m\right\}, \\
\mathrm{T}(\mathfrak{Q}, n) & =\min \left\{\mathrm{T}(\mathfrak{Q}, n ; \mathfrak{M}) \mid \mathfrak{M} \in \mathrm{Un}_{\mathcal{M}}(\mathfrak{Q})\right\}
\end{aligned}
$$

для любых $m, n \in \mathbf{N}$; если данных минимумов не существуют, то по определению полагаем, что $\mathrm{T}(\mathfrak{Q}, n ; m)=\mathrm{T}(\mathfrak{Q}, n)=\infty$. Функцию $\mathrm{T}(\mathfrak{Q}, n)$ назовем $T$-сложностью обхода класса $\mathfrak{Q}$ относительно класса $\mathcal{M}$; если из контекста ясно, о каком классе $\mathcal{M}$ идет речь, то в приведенном выражении указание на класс $\mathcal{M}$ опускаем.

Напомним, что под $r$-лабиринтом понимается конечный плоский мозаичный $(\{\Lambda\}, \bar{r} \cup\{\Lambda\})$-лабиринт, $r \in \mathbf{N}$; обозначим через $\widehat{\mathfrak{L}}(r)$ класс всех $r$-лабиринтов. Также напомним, что $r$-лабиринт $L$ называется $r$-деревом, если граф $\overline{G(L)}$ является деревом. Если метка любой дуги некоторого $r$-лабиринта $L$ равна символу $\Lambda$, то $L$ назовем неразмеченным $r$-лабиринтом. Ясно, что при любом $r$ класс всех неразмеченных $r$-лабиринтов является классом всех конечных плоских мозаичных лабиринтов, то есть совпадает с классом $\mathfrak{2}_{\mathrm{M}}^{\mathrm{K}}$. Положим также $\widehat{\mathfrak{Q}}(0)=\mathfrak{2}_{\mathrm{M}}^{\mathrm{K}}$.

Пусть $\mathfrak{A}$ - допустимый автомат для $\widehat{\mathfrak{Q}}(r)$, который при $r>0$ оснащен дополнительной функцией стирания и печатания символов из $\bar{r}$ на дугах лабиринтов из $\widehat{\mathfrak{L}}(r)$ : автомат $\mathfrak{A}$, двигаясь по таким лабиринтам, в любой момент дискретного времени меняет (и делает это обязательно) только метку дуги, которую выбирает в качестве направления своего дальнейшего продвижения, на некоторый символ из $\bar{r}$ (здесь не исключаем случай, когда автомат одну метку меняет на ту же самую); если $r=0$, то $\mathfrak{A}$ является автоматом (без печати) допустимым для $\widehat{\mathfrak{Q}}(0)=\mathfrak{2}_{\mathrm{M}}^{\mathrm{K}}$

Обозначим множество всех $r$-деревьев и множество всех неразмеченных $r$-деревьев, соответственно, через $\widehat{\mathfrak{L}}_{\mathrm{Z}^{*}}(r)$ и $\widehat{\mathfrak{L}}_{\mathrm{I}}(r)$. Также пусть $\widehat{\mathfrak{L}}_{0}(r)-$ множество всех неразмеченных $r$-лабиринтов и $\widehat{\mathfrak{Q}}_{*}(r)=\widehat{\mathfrak{Q}}(r)$. Тогда про автомат $\mathfrak{U}$, допустимый для $\widehat{\mathfrak{Q}}(r)$, говорим, что он является $\alpha$-универсальным, где $\alpha \in\left\{0, *\right.$, д $^{*}$, д\}, если $\mathfrak{A} \in \operatorname{Un}\left(\widehat{\mathfrak{Q}}_{\alpha}(r)\right)$. Положим $\mathbf{T}_{\alpha}(n, p, r)=\mathbf{T}\left(\widehat{\mathfrak{Q}}_{\alpha}(r), n ; p\right)$ для любого $\alpha \in\left\{0, *\right.$, д $^{*}$, д $\}$.

Теорема 11 ([60, 63]). При $n \geqslant 2$ имеют место соотношения:

$\mathbf{T}_{\partial}(n, 1,0)=\mathbf{T}_{\partial}(n, 2,0)=\mathbf{T}_{\partial}(n, 3,0)=\mathbf{T}_{\partial}(n, 1,1)=\infty u$

$$
2 n-3 \leqslant \mathbf{T}_{\partial}(n, p, r) \leqslant \begin{cases}(n-1)^{2} & \text { nрu } p=1, r \geqslant 2, \\ 3 n & \text { nрu } p=2,3, r \geqslant 1, \\ 2 n-3 & \text { nрu } p \geqslant 4, r \geqslant 0\end{cases}
$$

$\mathbf{T}_{\partial^{*}}(n, p, r)=\infty$ npu $p \in\{1,2,3\}, r \in\{0,1\} u$

$$
\mathbf{T}_{\partial^{*}}(n, p, r) \leqslant \begin{cases}3(n-1)^{2} & \text { nрu } p=1,2,3, r=2, \\ (n-1)^{2} & n p u p=1,2,3, r \geqslant 3, \\ 2 n & \text { nрu } p \geqslant 4, r \geqslant 0 ;\end{cases}
$$

$\mathbf{T}_{0}(n, p, 0)=\infty$ npu $p \geqslant 1, \mathbf{T}_{0}(n, 1,1)=\mathbf{T}_{0}(n, 2,1)=\infty u$

$$
2 n-3 \leqslant \mathbf{T}_{0}(n, p, r)< \begin{cases}2 n^{2} & \text { nрu } p=1,2,3, r \geqslant 2, \\ 4 n & \text { nрu } p \geqslant 4, r \geqslant 1\end{cases}
$$




$$
\begin{aligned}
& \mathbf{T}_{*}(n, p, r)=\infty \text { npu } p \geqslant 1, r \in\{0,1\} u \\
& \mathbf{T}_{*}(n, p, r) \leqslant \begin{cases}2 n^{3} & \text { npu } p=1,2,3,4, r=2, \\
2 n(n+1) & \text { npu } p \geqslant 5, r=2, \\
2 n^{2} & \text { npu } p \geqslant 1, r \geqslant 3 .\end{cases}
\end{aligned}
$$

Аналогично вводится понятие $\alpha$-универсальности для коллектива $\left(\mathfrak{U}_{1}, \mathfrak{A}_{2}\right)$, допустимого для класса $\widehat{\mathfrak{L}}(r)$. При этом предполагается, что если $\mathfrak{U}_{1}$ и $\mathfrak{A}_{2}$ одновременно делают шаг по одной и той же дуге, то метку ставит $\mathfrak{A}_{1}$. Через $\operatorname{At}_{\alpha}^{2}(p, r), \alpha \in\{0$, д\}, обозначим множество всех таких $\alpha$-универсальных коллективов, у которых любой из автоматов имеет $p$ состояний. Положим $\mathbf{T}_{\alpha}\left(\left(\mathfrak{H}_{1}, \mathfrak{H}_{2}\right), n\right)=\mathbf{T}\left(\widehat{\mathfrak{L}}_{\alpha}(r), n ;\left(\mathfrak{A}_{1}, \mathfrak{A}_{2}\right)\right)$ для любых $\alpha \in\left\{0\right.$, д\} и $\left(\mathfrak{A}_{1}, \mathfrak{A}_{2}\right) \in \mathrm{At}_{\alpha}^{2}(p, r), p \in \mathbf{N}$. $\mathrm{B}[63]$ показано, что при $n \geqslant 2$ для любого коллектива $\left(\mathfrak{A}_{1}, \mathfrak{U}_{2}\right) \in \mathrm{At}_{0}^{2}(p, r) \cup \mathrm{At}_{д}^{2}(p, r), p \geqslant 1, r \geqslant 0$, справедливо неравенство $\mathbf{T}_{\text {д }}\left(\left(\mathfrak{U}_{1}, \mathfrak{A}_{2}\right), n\right) \geqslant n-1$. Там же показано, что существуют $\left(\mathfrak{U}_{1}, \mathfrak{A}_{2}\right) \in \mathrm{At}_{д}^{2}(4,0)$ и $\left(\mathfrak{U}_{1}^{\prime}, \mathfrak{U}_{2}^{\prime}\right) \in \mathrm{At}_{0}^{2}(5,2)$ такие, что $\mathbf{T}_{\text {д }}\left(\left(\mathfrak{U}_{1}, \mathfrak{A}_{2}\right), n\right) \leqslant n-1$ и $\mathbf{T}\left(\left(\mathfrak{U}_{1}^{\prime}, \mathfrak{U}_{2}^{\prime}\right), n\right)<2 n$ при любом $n \geqslant 2$.

Конкретный прикладной аспект автоматного анализа лабиринтов обсуждается в [63]. В этой статье автоматы с печатью анализируют диаграммы Мура. У этих диаграмм входные символы закодированы элементами множества $\bar{k}, k \in \mathbf{N}$, выходные символы -- элементами множества $\bar{r}, r \in \mathbf{N}$, и одна вершина выбрана в качестве начальной (по существу диаграмма представляет собою регулярный нагруженный по дугам орграф степени $k$ ). Под автоматным $(k, r)$-лабиринтом или, короче, $(k, r)$-лабиринтом понимаем лабиринт, полученный из диаграммы Мура следующим способом:

- в качестве системы направлений возьмем множество входных символов;

- выходные символы вместе с пустым символом считаем метками дуг;

- некоторые выходные символы на дугах диаграммы сотрем (заменим эти символы на пустой символ $\Lambda$ ), и

- в качестве начальной вершины возьмем начальную вершину диаграммы.

Если стерты все выходные символы в диаграмме, то такой $(k, r)$-лабиринт называем $k$-графом. Иными словами, $(k, r)$-лабиринт - это регулярный степени $k$ инициальный $(\{\Lambda\}, \bar{r} \cup\{\Lambda\})$-лабиринт с множеством меток вершин $\{\Lambda\}$ и множеством меток дуг $\bar{k}, \mathrm{y}$ которого $[v]=\bar{k}$ для любой вершины $v$.

Обозначим множество всех $k$-графов через $P_{k}$, а множество всех сильно связных $k$ графов через $R_{k}$. Положим также

$$
H_{k, n}=\left\{L \in H_{k}|| V(L) \mid=n\right\}, \quad H_{k, n}^{*}=\left\{L \in H_{k}|| V(L) \mid \leqslant n\right\}, \quad H \in\{P, R\} .
$$

Допустимый для $(k, r)$-лабиринтов автомат с печатью имеет дополнительный выход $b$ для выдачи информационных сигналов со значениями из $\bar{p} \cup\{\Lambda\}, p \in \mathbf{N}$. Этот автомат, двигаясь по $(k, r)$-лабиринтам, в любой момент дискретного времени меняет (и это делается обязательно, причем не исключаются случаи, когда одна метка заменяется той же самой) только метку дуги, которую выбирает в качестве направления своего дальнейшего продвижения, на некоторый символ из $\bar{r} \cup\{\Lambda\}$ (печатание символа $\Lambda$ на некоторой дуге можно интерпретировать как стирание предыдущей метки). Обозначим через $\operatorname{At}(k, r, p)$ множество всех таких автоматов. 
Пусть $\mathfrak{A}-$ некоторый автомат из $\operatorname{At}(k, r, p)$ и $L-$ некоторый $(k, r)$-лабиринт. Автомат $\mathfrak{A}$ запускаем в $L$, поместив его в начальный момент $t=0$ в начальную вершину $v_{0}$ лабиринта $L$. Обозначим $b(t)$ значение выхода $b$ в момент времени $t$. Если существует $t_{0}=\min \{t \mid b(t) \neq \Lambda\}$, то пара Рез $(L, \mathfrak{A})=\left(t_{0}, b\left(t_{0}\right)\right)$, называется результатом работы $\mathfrak{A}$, и считается, что $\mathfrak{A}$ применим к $L$ и проверяет его за время $t_{0}$. В таком случае автомат $\mathfrak{A}$ называется также простым экспериментом для $L$; если он является таковым для каждого $L$ из класса $\mathfrak{L}$, то говорится, что он является простым экспериментом для $\mathfrak{L}$. Длиной простого эксперимента $\mathfrak{U}$ для $\mathfrak{L}$ называем минимальное $t \in \mathbf{N}_{0}$ такое, что $\mathfrak{U}$ проверяет любой лабиринт из $\mathfrak{Z}$ за время, не превосходяшее $t$. Простой эксперимент $\mathfrak{A}$ для $\mathfrak{L}$ называется безусловным, если для любых $L_{1}, L_{2} \in \mathfrak{L}$ имеет место равенство $\operatorname{Tr}_{\Sigma}\left(\mathfrak{A}, L_{1} ; t^{\prime}\right)=\operatorname{Tr}_{\Sigma}\left(\mathfrak{A}, L_{2} ; t^{\prime}\right)$, где $t^{\prime}-$ время, за которое $\mathfrak{A}$ проверяет хотя бы один из лабиринтов $L_{1}, L_{2}$; в противном случае $\mathfrak{A}$ есть условный эксперимент.

Пусть $\mathfrak{L} \subseteq P_{k}, \mathfrak{A}-$ простой эксперимент для $\mathfrak{L}, L \in \mathfrak{L}$. Эксперимент $\mathfrak{A}$ является

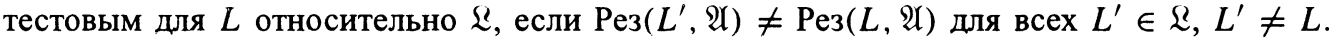
Эксперимент $\mathfrak{A}$ является диагностическим для $\mathfrak{L}$, если Рез $\left(L_{1}, \mathfrak{U}\right) \neq \operatorname{Pe}_{3}\left(L_{2}, \mathfrak{U}\right)$ для любых $L_{1}, L_{2} \in \mathfrak{Q}, L_{1} \neq L_{2}$.

Вершины $v$ и $v^{\prime} k$-графов $L$ и $L^{\prime}$ называются отличимыми автоматом за время $t$, если существует диагностический эксперимент $\mathfrak{U}$ для класса $\left\{L_{v}, L_{v^{\prime}}^{\prime}\right\}$ длины $t$. Вершины $v$ и $v^{\prime}$ называются отличимыми автоматом, если существует $t \in \mathbf{N}$ такое, что $v$ и $v^{\prime}$ отличимы автоматом за время $t$. Два $k$-графа называются отличимыми автоматом, если их начальные вершины отличимы автоматом.

Простой эксперимент $\mathfrak{A}$ для $\mathfrak{2}$ называется установочным для $\mathfrak{L}$, если для любых $L, L^{\prime} \in \mathfrak{Q}$ из равенства Рез $(L, \mathfrak{A})=\operatorname{Pе}\left(L^{\prime}, \mathfrak{A}\right)=(t, b(t))$ следует, что вершины $v_{t}$ и $v_{t}^{\prime}$ не являются отличимыми автоматом; вершина $v_{t}\left(v_{t}^{\prime}\right)$ - это вершина, в которую попадает автомат $\mathfrak{U}$ после проделанных первых $t$ шагов в $L\left(L^{\prime}\right)$.

Пусть $k \geqslant 1, m \geqslant n \geqslant 2, g(k, n)$ - минимальное время, достаточное для отличимости автоматом любой пары вершин любого $n$-вершинного $k$-графа, и $g(k, n, m)-$ минимальное время, достаточное для отличимости автоматом любой пары вершин такой, что первая из них принадлежит произвольному $n$-вершинному, а вторая - произвольному $m$-вершинному $k$-графу.

Теорема 12 ([63]). Для $g(k, n) u g(k, n, m)$ справедливы оченки

$$
\begin{aligned}
g(k, n) & = \begin{cases}2 n-4 & \text { при } k \geqslant 2, \\
n-1 & \text { в остальных случаях, }\end{cases} \\
g(k, n, m) & = \begin{cases}2 n-1 & \text { при } k \geqslant 2, m>n \geqslant 2, \\
2 n-3 & \text { при } k \geqslant 2, \\
n & \text { в остальных случаях. }\end{cases}
\end{aligned}
$$

Показано, что для любых $k \geqslant 2$ и $n \geqslant 4$ существует $n$-вершинный $k$-граф $L$ с попарно отличимыми автоматом вершинами такой, что отсутствует простой эксперимент, тестовый для $L$ относительно $[L]$, а также что отсутствует простой эксперимент, диагностический для $[L]$ (здесь $[L]-$ множество всех $k$-графов, которые получаются из $L$ изменением его начальной вершины).

Пусть $L \in R_{k, n}$ и $F \subseteq R_{k}$, где $F$ такое, что $L \in F$ и $L$ отличим автоматом от любого $L^{\prime} \in F, L^{\prime} \neq L$. Обозначим через $l_{F}(L)$ наименьшую длину безусловного простого эксперимента, тестового для $L$ относительно $F$. Пусть $l(L, r)=\max _{F} l_{F}(L)$, где максимум берется по всем указанным выше классам $F$ мощности $r$, а также $l(L)=l_{R_{k}^{\prime}}(L)$, где $R_{k}^{\prime}-$

2 Дискретная математика, т.15 №3 
класс попарно отличимых автоматом лабиринтов, который для любого лабиринта $L^{\prime} \in R_{k}$ содержит некоторый неотличимый от него лабиринт $L^{\prime \prime}$. Положим

$$
l(k, n, r)=\max \left\{l(L, r) \mid L \in R_{k, n}\right\}, \quad l(k, n)=\max \left\{l(L) \mid L \in R_{k, n}\right\} .
$$

Теорема 13 ([63]). Верны следующие соотночения.

Пусть $k \geqslant 2, n \geqslant 2 u r \geqslant 3$. Тогда

$$
l(k, n, r)=\frac{n(n+1)(k-1)}{2}+n
$$

$n p u r \geqslant n(k-1)+2 u$

$$
l(k, n, r) \sim \begin{cases}2 r(n-r) & \text { прu } 3 \leqslant r \leqslant n / 3, \\ (n+r)^{2} / 4 & \text { nрu } n / 3 \leqslant r \leqslant n, \\ r(n-r / 2 k) & \text { npu } n \leqslant r \leqslant n(k-1)+1\end{cases}
$$

npu $k, n, r \rightarrow \infty$.

Если $k \geqslant 2, r \geqslant 3$ и $r=o(n)$ при $n \rightarrow \infty$, mol $(k, n, r) \sim 2 n(r-1)$ nри $n \rightarrow \infty$.

$\Pi p u k \geqslant 2 u n \geqslant 2$

$$
l(k, n)=(1 / 2) n(n+1)(k-1)+n .
$$

Пусть $\mathscr{R}_{k, n}^{*}-$ класс всех $F \subseteq R_{k, n}^{*}$, которые состоят из попарно отличимых автоматом лабиринтов,

$$
\left(\mathscr{R}_{k, n}^{*}\right)_{r}=\left\{F \in \mathscr{R}_{k, n}^{*}|| F \mid=r \geqslant 2\right\}, \quad\left(\mathscr{P}_{k, n}^{*}\right)_{r}=\left\{F \subseteq P_{k, n}^{*}|| F \mid=r \geqslant 2\right\} .
$$

Обозначим через $v(F)(h(F))$ наименьшую длину условного простого эксперимента, диагностического (установочного) для $F \in \mathscr{R}_{k, n}^{*}\left(F \subseteq P_{k, n}^{*}\right)$. Также положим

$$
\begin{aligned}
v(k, n, r) & =\max \left\{v(F) \mid F \in\left(\mathscr{R}_{k, n}^{*}\right)_{r}\right\}, \\
(h(k, n, r) & \left.=\max \left\{h(F) \mid, F \in\left(\mathscr{P}_{k, n}^{*}\right)_{r}\right\}\right),
\end{aligned}
$$

$v(k, n)=v\left(\hat{R}_{k, n}^{*}\right)$, где $\hat{R}_{k, n}^{*}-$ класс попарно отличимых автоматом лабиринтов, который для каждого лабиринта $L \in R_{k, n}^{*}$ содержит некоторый неотличимый от него автоматом лабиринт $L^{\prime}$, и $h(k, n)=h\left(P_{k, n}^{*}\right)$. В [63] показано, что для функций $v(k, n, r)$ и $h(k, n, r)$ $(v(k, n)$ и $h(k, n))$ справедливы утеерждения (утверждение), сформулированные (сформулированное) в теореме 13 для функции $l(k, n, r)(l(k, n))$.

Пусть $L \in P_{k}$. Простой эксперимент $\mathfrak{U}$ для $[L]$ называется установочным для $L$, если для любых $v, v^{\prime} \in V(L)$ из Рез $\left(L_{v}, \mathfrak{A}\right)=\operatorname{Pе}\left(L_{v^{\prime}}, \mathfrak{U}\right)=(t, b)$ следует, что $v_{t}=v_{t}^{\prime}$, где $v_{t}\left(v_{t}^{\prime}\right)$ - вершина, в которую попадает $\mathcal{U}$ после $t$ шагов в лабиринте $L_{v}\left(L_{v^{\prime}}\right)$. Лабиринт $L$ называется ориентируемым, если для него существует установочный эксперимент. Обозначим через $H_{k, n}$ множество всех $K \in P_{k, n}$ с занумерованными вершинами и с невыделенной вершиной в качестве входа, через $S_{k, n}-$ множество всех ориентируемых лабиринтов из $H_{k, n}$ и через $E_{k, n}-$ класс всех попарно неизоморфных (в смысле перенумерации вершин) лабиринтов из $S_{k, n}$. В [63] показано, что

$$
\left|S_{k, n}\right| \sim n^{k n}, \quad\left|E_{k, n}\right| \sim n^{k n} / n !, \quad\left|S_{k, n}\right| /\left|H_{k, n}\right| \rightarrow 1,
$$

при $k \geqslant 2, n \geqslant 2$ и $k+n \rightarrow \infty$. 
В [48] в качестве основного объекта исследования выступает один подкласс класса всех автоматных $(k, r)$-лабиринтов (см. [63-65]), а именно, множество всех автоматных лабиринтов. Под автоматным лабиринтом понимаем $k$-граф, у которого все вершины достижимы из его начальной вершины; обозначим через $\Gamma_{n}$ класс всех $n$-вершинных автоматных лабиринтов.

Пусть $L-$ некоторый автоматный лабиринт, $v \in V(L)$ и $\alpha \in(\bar{k})^{*}$. Если существует путь

$$
v_{0}=v, e_{1}, v_{1}, e_{2}, v_{2}, \ldots, v_{n-1}, e_{n}, v_{n}
$$

такой, что $\left|e_{1}\right|\left|e_{2}\right| \ldots\left|e_{n}\right|=\alpha$, то вершину $v_{n}$ обозначим через $v \alpha$, а сам путь - через $\mathfrak{p}(v, \alpha)$. Два автоматных лабиринта $L_{v_{0}}$ и $L_{v_{0}^{\prime}}^{\prime}$ являются изоморфными, $L_{v_{0}}=L_{v_{0}^{\prime}}^{\prime}$, если существует биекщия $\varphi: V\left(L_{v_{0}}\right) \rightarrow V\left(L_{v_{0}^{\prime}}^{\prime}\right)$ такая, что $\varphi\left(v_{0}\right)=v_{0}^{\prime}$ и $\varphi(v x)=\varphi(v) x$ для любого $x \in \bar{k}$.

У допустимых автоматов класса $\Gamma_{n}$ предполагается наличие дополнительного выхода для выдачи информационных сигналов из множества $\{\Lambda\} \cup \overline{2}$, а также предполагается, что он в состоянии не больше одного раза ставить метку в каждую вершину лабириіта, в котором находится, а также регистрировать наличие метки в текущей вершине.

Пару слов $W=\{\alpha, \beta\}, \alpha, \beta \in(\bar{k})^{*}$, назовем экспериментом, различаюшим автоматные лабиринты $L_{v_{0}}$ и $L_{v_{0}^{\prime}}^{\prime}$, если $\left(v_{0} \alpha=v_{0} \beta \wedge v_{0}^{\prime} \alpha \neq v_{0}^{\prime} \beta\right) \vee\left(v_{0} \alpha \neq v_{0} \beta \wedge v_{0}^{\prime} \alpha=v_{0}^{\prime} \beta\right)$

Для любых слов $\alpha$ и $\beta$ в некотором алфавите пишем $\alpha \leqslant \beta$, если $\alpha$ - начало слова $\beta$. Пусть теперь $W=\left\{\alpha_{1}, \alpha_{2}\right\}-$ некоторый эксперимент, различающий автоматные лабиринты $L_{v_{0}}$ и $L_{v_{0}^{\prime}}^{\prime}$. Если $\alpha_{1} \nless \alpha_{2}$ и $\alpha_{2} \nless \alpha_{1}$, то эксперимент назовем кратным и под его сложностью понимаем число $C(W)=\left|\alpha_{1}\right|+\left|\alpha_{2}\right|$, где $\left|\alpha_{i}\right|-$ длина слова $\alpha_{i}, i=1,2$. Если $\alpha_{1} \leqslant \alpha_{2}$ или $\alpha_{2} \leqslant \alpha_{1}$, то эксперимент назовем простым и под его сложностью понимаем число $C(W)=\max \left\{\left|\alpha_{1}\right|,\left|\alpha_{2}\right|\right\}$. Эксперимент минимальной сложности называется кратчайшим.

В случае кратного эксперимента построим автоматы $\mathfrak{A}_{1}$ и $\mathfrak{A}_{2}$, которые в паре фу: $1:-$ ционируют следующим способом. Сначала запускается автомат $\mathfrak{A}_{1}$ в лабиринте $L_{v_{0}}$. $\mathrm{OH}_{\mathrm{H}}$ проходит путь $\mathfrak{p}\left(v_{0}, \alpha_{1}\right)$ и ставит метку в вершине $v_{0} \alpha_{1}$. Затем запускается автомат $\mathfrak{U}_{2}$ в $L_{v_{0}}$, который проходит путь $\mathfrak{p}\left(v_{0}, \alpha_{2}\right)$, и если обнаруживает метку в вершине $v_{0} \alpha_{\overline{2}}$, то выдает информационный сигнал 1, в противном случае выдает сигнал 2. В случае простого эксперимента можем построить один автомат, который в лабиринте $L_{v_{0}}$ проходит путь $\mathfrak{p}\left(v_{0}, \alpha_{1}\right)$, ставит метку в вершине $v_{0} \alpha_{1}$, затем проходит путь $\mathfrak{p}\left(v_{0} \alpha_{1}, \alpha_{3}\right)$, где $\alpha_{3}-$ такое слово, что $\alpha_{2}=\alpha_{1} \alpha_{3}$, и если обнаруживает метку в вершине $v_{0} \alpha_{2}$, то выдает информационный сигнал 1, в противном случае выдает сигнал 2. Ясно, что таким способом можно различать данные автоматные лабиринты, а сложность эксперимента фактически определяет необходимое время для различения лабиринтов в данном эксперименте.

В [48] показано, что любые два неизоморфных автоматных лабиринта различаются простым или кратным экспериментом. Основные результаты работы [48] сформулированы в виде следующих двух теорем. Пусть $k \geqslant 1$ и $n \geqslant 2$.

Теорема 14. Сложность кратчайшего эксперимента, различающего любые два неизоморфных графа из класса $\Gamma_{n}$, не превосходит $C(n, k)$, где

$$
C(n, k)= \begin{cases}2 n-4, & \text { если } k \geqslant 2, n \geqslant 3, \\ n, & \text { в остальных случаях }\end{cases}
$$

причем указанные оченки неулучшаемы. 
Теорема 15. Пусть $m>n$. Сложность кратчайшего эксперимента, различающего любые два графа $G \in \Gamma_{n}$ и $H \in \Gamma_{m}$, не превосходит $C(n, m, k)$, где

$$
C(n, m, k)= \begin{cases}2 n-2, & \text { если } k \geqslant 2, \\ n, & \text { если } k=1,\end{cases}
$$

причем указанные оченки неулучшаемы.

В [48] приведены лабиринты, которые различаются только простыми или только кратными экспериментами, а также и следующее утверждение, которое понижает оценку теоремы 14.

Теорема 16. Если для графов $G, H \in \Gamma_{n}$, существуют только простые различающие эксперименты, то сложность кратчайшего эксперимента не превосходит $n$ и эта оченка неулучиаема.

\section{5. Вместо заключения}

Как видно из изложенного, изучение поведения автоматов в лабиринтах привело к созданию и утверждению важного направления в теории автоматов. Разработан понятийный аппарат, позволяющий решать имеющиеся проблемы и формулировать новые. Много важных проблем решено, но некоторые известные проблемы требуют своего решения. Многие из них приведены в нашем обзоре. Заметим, что направление, посвященное автоматам в лабиринтах, развивалось благодаря в основном усилиям ученых Германии, России, Сербии и Черногории, США и Японии. С сожалением можно отметить, что в последние несколько лет ощущается своеобразное затишье в исследуемой области. Это может отчасти объясняться трудностью самих проблем. Следует также иметь в виду, что некоторые центры, в которых развивалось это направление перестали существовать. В основном новые результаты в последние годы получены школой, объединяющей ученых Московского государственного университета им. М. В. Ломоносова. Частью этой школы можно считать и группу ученых Белградского университета, среди которых находятся и авторы данной работы.

\section{Список литературы}

1. Asser G., Bemerkungen zum Labyrinth-Problem. Elektron. Inform.-verarb. Kybernetik (1977) 13, 203-216.

2. Blum M., Hewitt C., Automata on a 2-dimensional tape. In: FOCS 1967, pp. 155-160.

3. Blum M., Sakoda W., On the capability of finite automata in 2 and 3 dimensional space. In: FOCS 1977, pp. 147-161.

4. Blum M., Kozen D., On the power of the compass. In: FOCS 1978, pp. 132-142.

5. Budach L., Two pebbles don't suffice. Lect. Notes Comput. Sci. (1981) 118, 578-589. (1981).

6. Bull M., Hemmerling A., Finite embedded trees and simply connected mazes cannot be searched by halting finite automata. J. Inf. Process. Cybern. (1990) 26, 65-73.

7. Coy W., Of mice and maze. Elektron. Inform.-verarb. Kybernetik (1978) 14, 227-232.

8. Ejsmont M., Problems in labyrinths decidable by pebble automata. Elektron. Inform.-verarb. Kybernetik (1984) 20, 623-632. 
9. Graw B., On tape complexity classes and Savitch mazes. Elektron. Inform.-verarb. Kybernetik (1981) 17, 501-510.

10. Habasinski Z., Karpinski M., A Codification of Blum-Sakoda 7 Pebbles Algorithm. Pr. Inst. Podstaw Inf. Pol. Akad. Nauk 448, 1981.

11. Hemmerling A., Kriegel K., On searching of special classes of mazes and finite embedded graphs. Lect. Notes Comput. Sci. (1984) 176, 291-300.

12. Hemmerling A., 1-pointer automata searching finite plane graphs. Z. Math. Logik Grundlagen Math. (1986) 32, 245-256.

13. Hemmerling A., Remark on the power of compass. Lect. Notes Comput. Sci. (1986) 233, 405-413.

14. Hemmerling A., Three-dimensional traps and barrages for cooperating automata. Lect. Notes Comput. Sci. (1987) 278, 197-203.

15. Hemmerling A., Normed two-plane traps for finite systems of cooperating compass automata. J. Inf. Process. Cybern. (1987) 28, 453-470.

16. Hemmerling A., Labyrinth problems. Labyrinth-searching abilities of automata. Teubner, Leipzig, 1989.

17. Hemmerling A., Pebble automata in labyrinths with rotation systems. Z. Math. Logik Grundlagen Math. (1991) 37, 453-466.

18. Hoffmann F., One pebble does not suffice to search plane labyrinths. Lect. Notes Comput. Sci. (1981) 117, 433-444.

19. Hoffmann F., 1-Kiesel-Automaten in Labyrinthen. Report R-Math-06/82, 1982, Adw. der DDR, Berlin.

20. Inoue K., Takanami I, Nakamura A., A note on two-dimensional finite automata. Inf. Process. Lett. (1978) 7, 49-52.

21. Inoue K., Nakamura A., Two-dimensional finite automata and unacceptable functions. Int. J. Comput. Math. (1979) 7, 207-213.

22. Inoue K., Takanami I., A note on decision problems for three-way two-dimensional finite automata. Inform. Process. Lett. (1980) 10, 245-248.

23. Kilibarda G., On the minimum universal collectives of automata for plane labyrinths. Discrete Math. Appl. (1993) 3, 555-586.

24. Kinber E. B., Three-way automata on rectangular tapes over a one-letter alphabet. Inform. Sci. (1985) 35, 61-77.

25. Kriegel K., Universelle 1-Kiesel-Automaten für k-komponentige Labyrinthe. Report R-Math-04/84, 1984, Adw. der DDR, Berlin.

26. Kozen D., Automata and planar graphs. In: Fundamentals of Computation Theory. Akademie, Berlin, 1979, pp. 243-254.

27. Kudryavtsev V. B., Ushchumlich Sh., Kilibarda G., The behaviour of automata in labyrinths. Discrete Math. Appl. (1993) 3, 1-28.

28. Milgram D. L., Rosenfeld A., Array automata and array grammars. In: IFIP Congress 71 . NorthHolland, Amsterdam, 1971, pp. 69-74.

29. Milgram D. L., A region crossing problem for array-bounded automata. Inform. and Control (1976) 31, 147-152.

30. Mylopoulos J., On the recognition of topological invariants by 4-way finite automata. Computer Graphics and Image Processing (1972) 1, 308-316.

31. Mylopoulus J., On the application of formal languages and automata theory to pattern recognition. Pattern Recognition (1974) 4, 37-51. 
32. Rollik H. A., Automaten in planaren Graphen. Acta Inf. (1980) 13, 287-298.

33. Savitch W., Relations between nondeterministic and deterministic tape complexities. J. Comput. System Sci. (1970) 4, 177-192.

34. Savitch W., Maze recognizing automata and nondeterministic tape complexity. J. Comput. System Sci. (1973) 7, 389-403.

35. Shah N. A., Pebble automata on arrays. Computer Graphics and Image Processing (1974) 3, 236246.

36. Szepietowski A., A finite 5-pebble-automaton can search every maze. Inform. Process. Lett. (1982) 15, 199-204.

37. Szepietowski A., On searching plane labyrinths by 1-pebble automata. Elektron. Inform.-verarb. Kybernetik (1983) 19, 79-84.

38. Szepietowski A. Remarks on searching labyrinths by automata. Lect. Notes Comput. Sci. (1983) 158, 457-464.

39. Pultr A., Úlehla J., On two problems of mice. Rend. Circ. Mat. di Palermo (1982) 31, 249-262.

40. Анджанс А. В., О возможностях автоматов при обходе одномерных областей. Латв. матем. ежегодник (1983) 27, 191-201.

41. Анджанс А. В., Возможности автоматов при обходе плоскости. Проблемы передачи информачии (1983) 19, №3, 78-89.

42. Анджанс А. В., Сложность определения автоматом его расположения относительно замкнутого контура. В кн.: Математическая логика, математическая лингвистика и теория автоматов. Калининский гос. ун-т, Калинин, 1983, с. 88-90.

43. Анджанс А. В., О возможностях автоматов при обходе пространства. В кн.: Межвуз. сб. работ по теории автоматов, алгебре и теории чисел. Рига, 1984, с. 3-17.

44. Анджанс А. В., Поведение детерминированных и вероятностных автоматов в лабиринтах. Дисс. канд. физ.-мат. наук, Рига, 1987.

45. Грунская В. И., О взаимодействии автоматов типа хищник-жертва. Дипломная работа, МГУ, 1988.

46. Грунская В. И., О динамическом взаимодействии автоматов. В кн.: Математическая кибернетика и ее приложения к биологии. Изд-во МГУ, Москва, 1987, с. 8-18.

47. Грунская В. И., О контекстной зависимости множеств протоколов автоматов в геометрических средах. В кн.: Труды международной конферениии по интеллектуальным системам. Москва, 1994.

48. Грунский И. С., Олейник Р. И., Об отличимости инициальных автоматных лабиринтов конечными автоматами. Интеллектуальные системы (1999) 4, 273-283.

49. Килибарда Г., Об обходе конечных лабиринтов системами автоматов. Дискретная математика (1990) 2, №2, 71-81.

50. Килибарда Г., О сложности автоматного обхода лабиринтов. Дискретная математика (1993) 5, №3, 116-124.

51. Килибарда Г. О минимальных универсальных коллективах автоматов для плоских лабиринтов. Дискретная математика (1994) 6, №4, 133-153.

52. Килибарда Г., Ушчумлич Ш., О лабиринтах-ловушках для коллективов автоматов. Дискретная математика (1993) 5, №2, 29-50.

53. Килибарда Г., Ушчумлич Ш., О задаче синтеза для автоматов в одном классе лабиринтов. FILO-MAT (1995) 9, 743-751. 
54. Килибарда Г., Кудрявцев В. Б., Ушчумлич Ш., Независимые системы автоматов в лабиринтах. Дискретная математика (2003) 15, №2, 3-39.

55. Клини С., Представление событий в нервных сетях и конечных автоматах. В кн.: Автоматы. ИЛ, Москва, 1956, с. 15-67.

56. Кудрявцев В. Б., Ушчумлич Ш., Килибарда Г., О поведении автоматов в лабиринтах. Дискретная математика (1993) 4, №3, 3-26.

57. Кудрявцев Г. Ю., О времени решения лабиринтной задачи конечными автоматами. Сб. научн. трудов МЭИ (1987) 138, 14-18.

58. Кудрявцев Г. Ю., О времени обхода лабиринтов без циклов конечными автоматами. В кн.: Материалы 2-го Всесоюзиого семинара по дискретной математике и ее приложениям. Изд-во МГУ, Москва, 1988, с. 202-208.

59. Кудрявцев Г. Ю., О сложности конечных автоматов, решающих задачу о лабиринте. Деп. ВИНИТИ, 4.05.88, 3430-В88.

60. Кудрявцев Г. Ю., О сложности конечных автоматов, решающих лабиринтную задачу. В кн.: Алгебро-логические колструкции. Межвуз. темат. сб. трудов. Калининский гос. ун-т, Калинин, 1989, c. 68-71.

61. Кудрявцев Г. Ю., О времени обхода лабиринтов конечными автоматами. В кн.: Межсвуз. сб. трудов. Саратовский гос. ун-т, Саратов, 1989, с. 95-105.

62. Кудрявцев Г. Ю., О сложности экспериментов с конечными графами. Деп. ВИНИТИ 20.11.89, 6961-B89.

63. Кудрявцев Г. Ю., О времени решения лабиринтиых задач конечными автоматами. Дисс. канд. физ.-мат. наук, Саратов, 1990.

64. Кудрявцев Г. Ю., Об отличимости вершин автоматных лабиринтов конечными автоматами. Дискретная математика (1991) 3, №4, 143-152.

65. Кудрявцев Г. Ю., О длине тестовых автоматно реализуемых экспериментов с автоматными лабиринтами. Дискретная математика (1992) 4, №3, 86-100.

66. Кудрявцев Г. Ю., О времени решения лабиринтных проблем конечными автоматами. Докл. РАН (1992) 326, 601-604.

67. Курдюмов Г. Л., Коллектив автоматов с универсальной проходимостью. Проблемы передачи информачии (1981) 17, №4, 98-112.

68. Стаматович Б., Распознавание спечиальных классов л-лабиринтов автоматами. Дисс. докт. физ.-матем. наук. Матем. ф-т, Белград, 1999.

69. Стаматович Б., Распознавание односвязных цифр автоматом. Интеллектуальные системы (1998) 3, 291-305.

70. Стаматович Б., Распознавание двусвязных цифр коллективами автоматов. Интеллектуальные системы (1998) 4, 321-337.

71. Феллер В., Введение в теорию вероятностей и ее приложения, т. 1, Мир, Москва, 1984.

Статья поступила 20.08.2003. 